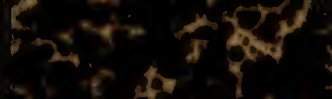

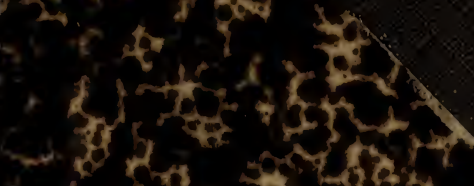

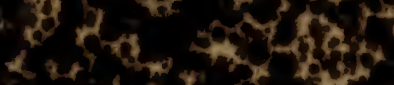

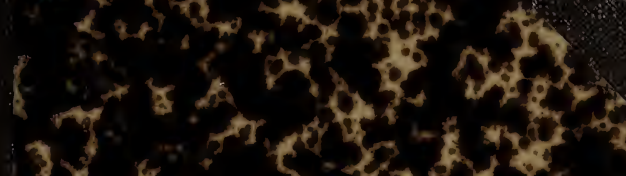

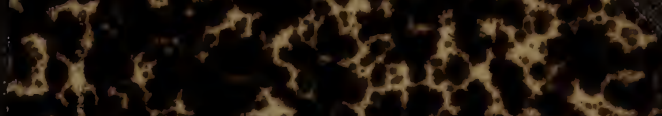
सर

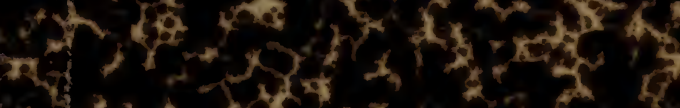

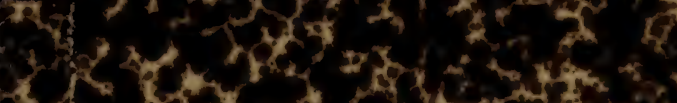

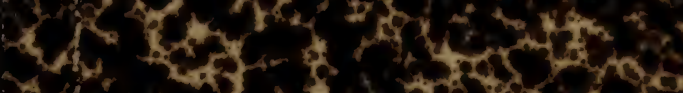

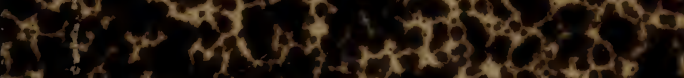

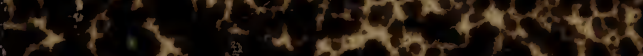

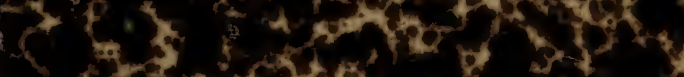

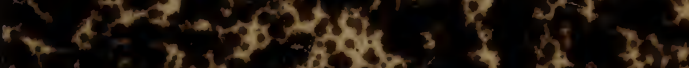

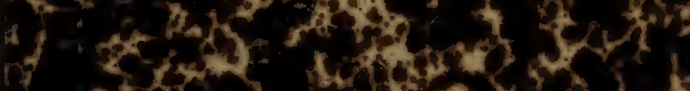

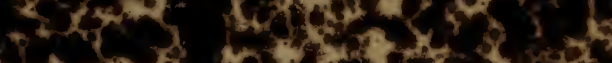

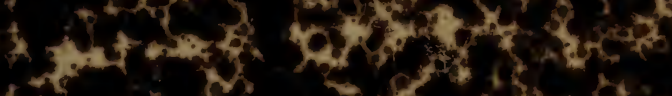

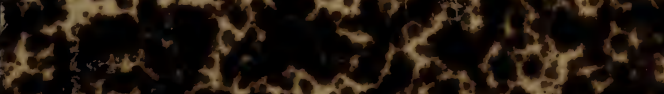
है

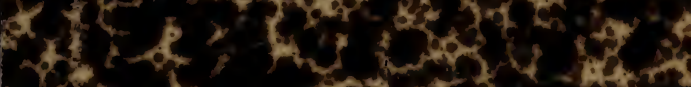
at 1 .

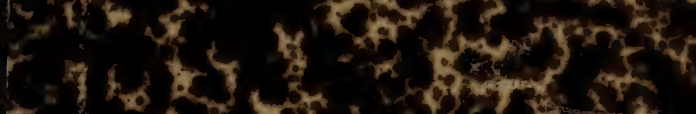

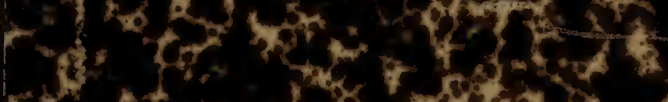

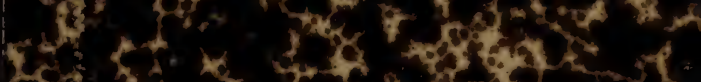

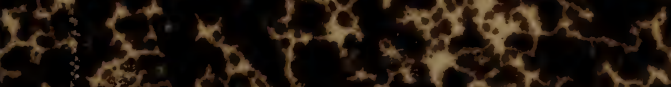

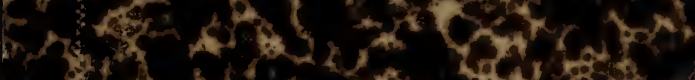
* a h

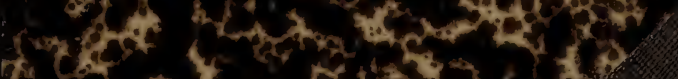

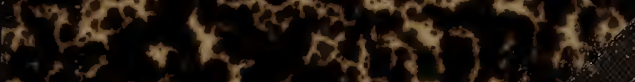

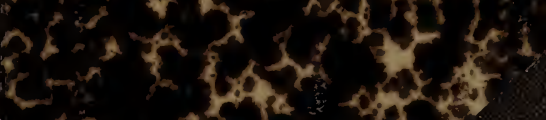
$2 x^{2}+x^{2}+2 x+125$

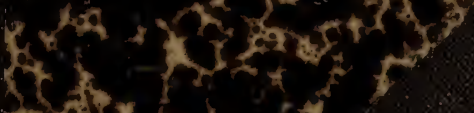


$M \mathrm{H}$
$\mathrm{M} 36$

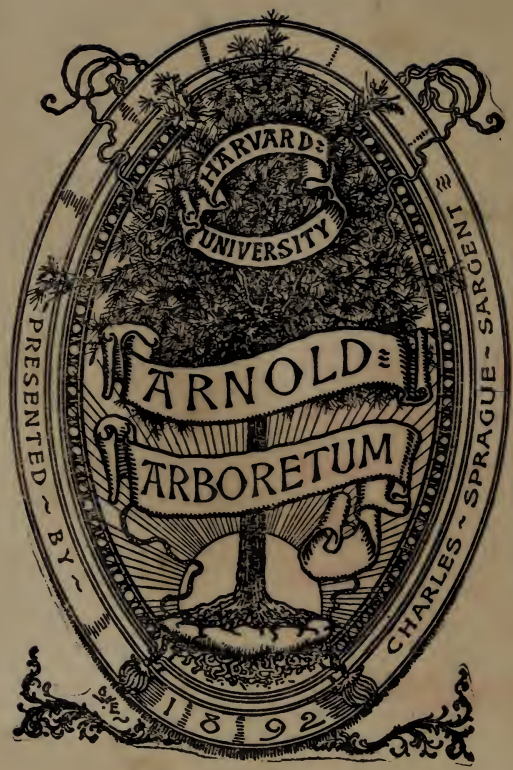




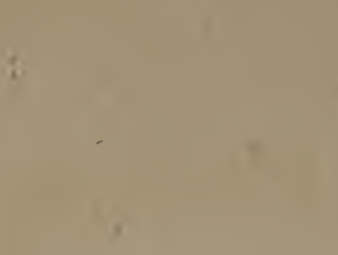

$$
\text { , }
$$
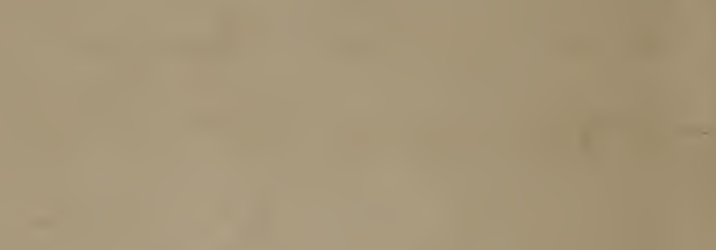

(1)
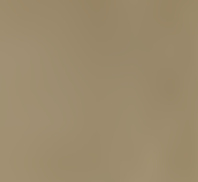

15.

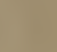

$-$
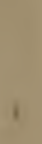

$-$

$$
=
$$




\title{
(jefammelte Padhiddten
}

\author{
uiber ben
}

\section{DEacuffarifiden Sififtbaum}

Wott

\section{Ernft $\mathfrak{B}$ Bllbelm Martius}

ber Regensburgifchen botanifhen Stefellichaft ordentlichem Mitgliede.

Mit einer illuminirten Rupfertafel.

$$
\mathfrak{E} \mathfrak{l} \mathfrak{a} \mathfrak{n} \mathfrak{g} \mathfrak{e} \mathfrak{n}
$$

in ber 2 Saltherichen $\mathfrak{B}$ udbjandlung. 1792. 


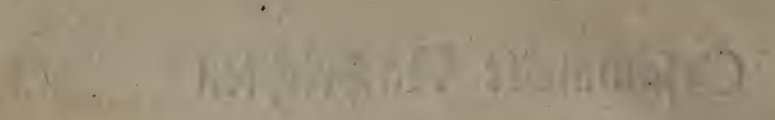

$x$

the wh

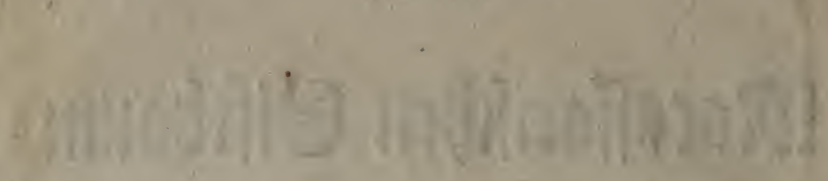

$$
\pm=
$$

\section{: 1}

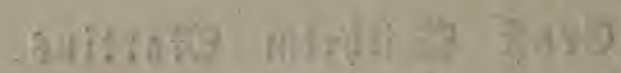

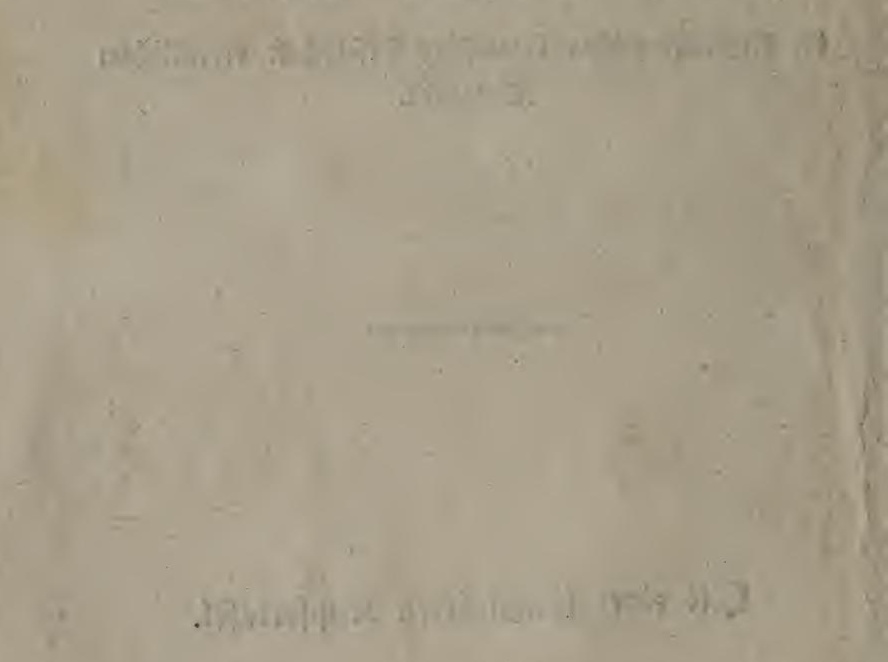

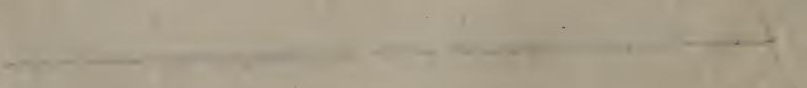

\section{(4) $x+301 \% ?$}

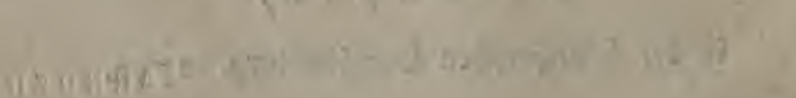

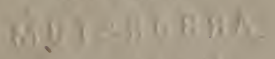
Ihoxy youst

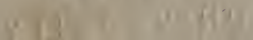




\title{
Sr. $\mathfrak{B}$ ohlgebohrnen
}

bem

\author{
Sอ $\mathfrak{x} \mathfrak{n}$
}

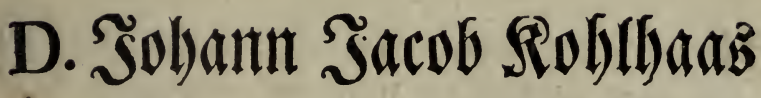

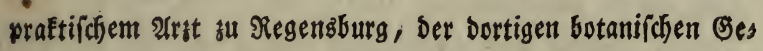
Fellichaft Práfibenten, und ber gelebrten sereellichaften ău

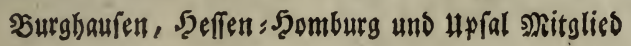

wismet

\section{Diefe $\mathfrak{B}$ I a t te}

als ein geringes Denfmal feiner Scodjadjtung

Der

Derfaffer. 

D Neníf, Dem Dic Natur, Den \&ebensbaüch) zu frifen,

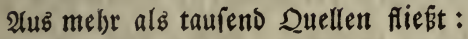

D fielse bich wobl vor, weil oft aus ibren \$rtuften Sich audf ein tóblich sifft ergieft.

(S) iftarten, bie entweber innerlich ober åuferlic) beigebrad)t, bie feften unb fuiffigen Sheile bes

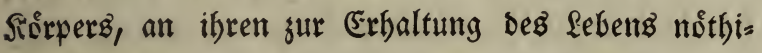
gen Serridtungen hinbern, tmb ůber furg ober lang ben Tod nach fich) zieben, fommen in ben brei Rieis c)en ber গatur båufig vor. Dergleichen finb aus Den Nineralreiche Irfenif, werichiebene Bubereitun= gen aus Luecfillber, Slen und Spief̧glang; fo wie aud) Die farfen Nineralfâturen.

Eben fo tobliche Subftansen liefert auch bas Ifier = und Pflangenreid), welche sffers, bejonders in entferntern Giegenden unjers Frbbobenz, um beswil= len befto gefåbrlicher finb, weil man nicht immer if = ren (chåblichen sisirkungen, theils aus mangelnber

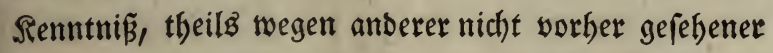
Bufålle augigumeichen im Stande ift. So erregt junt Seifpiel im Sbierreidhe baz ङift ber Edylangen mit ei ner furdhtbaren Befdrwindigfeit tod lid)e 2nfålle; unt fo wie Das Pflanjenreich viele beilfame Mittel bar: 
bietet: fo erfengt er aber aud) cbenfalls ill groferer 211 . gahl gefäbrliche (iiftarten, Deren einige corrofivifa find, und Entgundungen verurfachen, aubre bas Slut unb bie Feudhtigfeiten verberben uno verjebren, andre

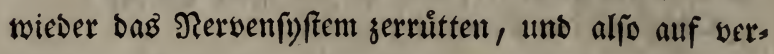
f́chicbene 2 seife ben Rebensfaben verfúrjen, wie ;. $\mathfrak{b}$.

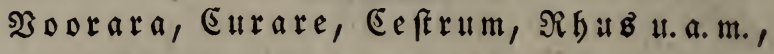
von welchen Gerwåchfen ich, elge id) weiter gelge, eini= ge ber merfwurbigften juerft anführen-werbe.

Die febr giftige $\mathfrak{B}$ oorara, ober $\mathfrak{X}$ urali, wie fie bie auf ben offtichen Theil won Guiana woh) nenden भrrawaffen menuen, ift cin Ranfengewåd)s, weldjes von einigen aud) $\mathfrak{b} u(d) t a \mathfrak{u}$ genennt wirb, weil es fid) Dem 2 (n) (d)ein nach, gleich Dem Taumerf an einem Sdhiffe, an ben ůbrigen in ber शåke bes finblid)en biåumen oft obute $\mathfrak{b}$ låtter uno Aefte bin= auf fdhlingt, aber mefrmals von ber Dicfe eints Arms ober cines Seins ift. - 2 us biefem bies

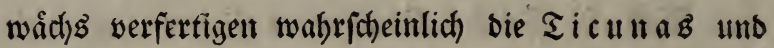

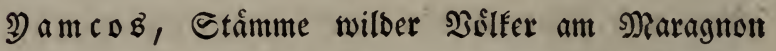
- der Amagonenfluffe, ibs beruedtigtes $\mathfrak{P}$ feilgift, weld)es burch ben f̧errn von Eondamine bes fannt worben ift. (S. Sancrofts Naturgejhichte von Öıtiana 1769. S. I75.) Sie nebmen nåm=

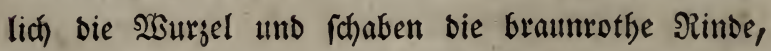
bie etwa fo bick als ein Nagel ant Finger ift,

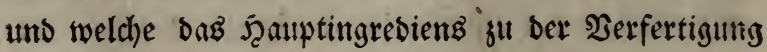
Des Giftes if, bavon ab. Diefe Rimbe wirb podann- 


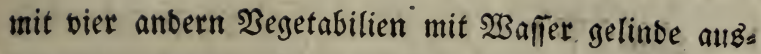

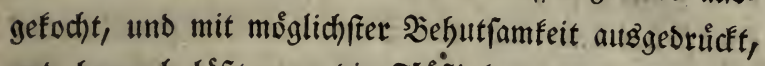

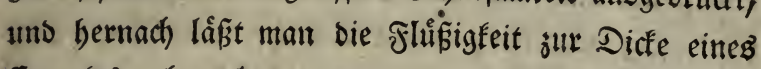
Extraftis abraudjen.

Scieber ift audh nod) bie (E trare zat jáblen, aus ber bie Raffern am Dronoque' iffr Gift verfertigen, und welde nach bem Serid)t Deb fren. von $P_{a} u$ w eine Riane (fletternber Etraud)) iff, tho vielfeid)t biel mit bem vorfergeheriden (jerwadds überein hat.

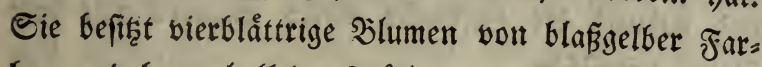
be, unt bernach fleine fruid,te von ber Geftalt einer Sobne, beren srei beifammen in einer birnformigen frudht enthalten fint. Hebrigens fheint bies Gies wâd): mit ben Siriolbenaugen (Strychnos Nux vomica L.) in naber פerwanbidyaft ful fteben.

Ein anbres fohăbliches Gewåchs ift ber auf ben

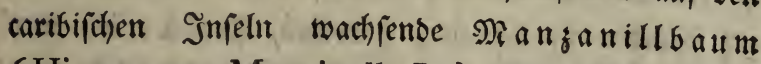
(Hippomane Mancinella L.) (Er wâd)ft bis jutt

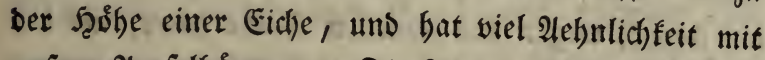
unern 2lepfelbåumen. Die Siinde ift glatt, braun,

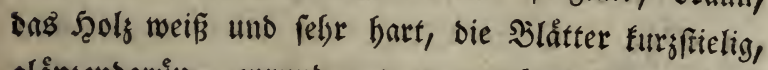
glånzenbgrůn, eyruno, von gezacFten Ranbe, ber

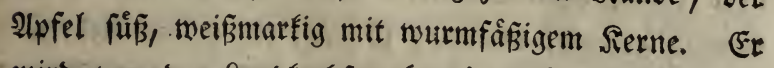
wirb won ben Lanbfiebfen (ruricolis) gefreffen, bie bavon giftig werben. In ber Sinve unb bem 2 pfel

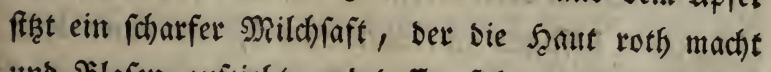
uno Slafen aufjiebt, unb beffen fids bie Eariben ebes I 2 bem 
bem gleidjfalls zum $\mathfrak{B}$ ergiften ifrer $\$$ Peile bebtenten: Somft bat man aud) Den Dunft bes Manjanilfbaumes für giftig gebalten, alfein ber Şr. von Jacqui werfichert (Select. ftirp. americ. hiftor. p. 25r.) bafis er es aus (Erfabrung nidyt fo befunden babe, nuch babe er bemerft, baf ber von siefem Saum abs trâufeltube Siegen nicht giftig fey; Daf man folg:

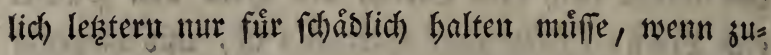

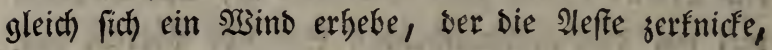
uns baburch bic Giftmildy Diefes \$aumes mit bem গiegen vermifche.

Son Der Gattung beb গi finb stei Irten als giftige befannt; Die erfte bahin geborige ?lat ift:

Der Firni $\mathfrak{b} \mathfrak{b} \mathfrak{m}$ (Rhus Vernix L.) E⿺ ift eitr berwobner ber fetchten, nafien und furmpfict)= ten 2 Bâloer in Canada, Earolina, Penfilvanien uno Japan. Er breitet feitre atweige febr weit aus, uns bat fowohl wegen bez fa)nellen $2 \mathfrak{s a c h}$ sthumb, als ber

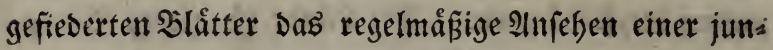
gen Eface. Seime Slluthe bat eitren angenehmen

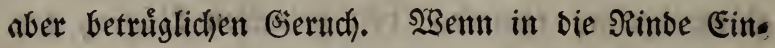
(ch) nitte gentacht merben, fo tritt ein gelber flebrich) ter und mildhid)ter Saft beraus, weld)en bie Ehine: fe: zum Racfiren verwenden. Diefer Saft bat wie bas Şolf cinen beftigen fiutfenden (jerud), und einen ball

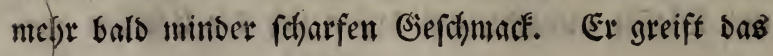
Bifen 


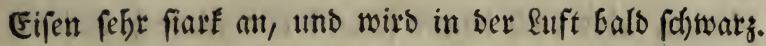
Das Bjelangen beflelben an bie Şaut und 2ugen, fo wie auch) bie Ausbuinfung bes Saumes, verurfacht

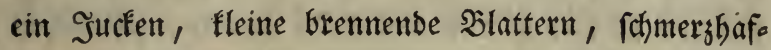

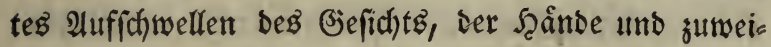
len Des gangen Riorpers. Die djinefifhen Arbeiter, weldje ben Firnif́ aus bem baum zieben, befommen in 24 Ctunben ein Siothlauf ůber ben ganjen leib, woburch fie bas Anfehgen eines 2uşåţigen erhalten, wobei ifnen audh nach unb nach bie స̧aut berftet, aus beren Spalten fich fobann viel fharfes Serum ergiefst, endich) trocknet aber bie f̧aut wieber ab,

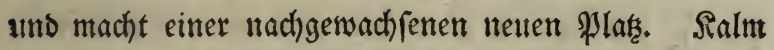
bemerft aber in feiner Reifebeichr. Th. 2. S.228. u. f. baß̃ nicht ein jeber ben angefůfrten Zufâllen ausges feţt fen, fonbern mandje gegen bas Gift siefes Dalts mes recht nothfeft wåken.

Eine anbere gefâfrliche $\mathfrak{A n t}$ bes $\Re \mathfrak{h}$ แ (b) if $t \mathfrak{b} a \mathfrak{m}$, ober nad) (Glebitfd) ridfiger, ber grofle breiblåtrige (Eanabifde Giftres benftra $\mathfrak{a} \mathfrak{d}$ (Rhus Toxicodendron L.) Die $2 \mathfrak{s u r =}$ zel biefés Strauches gleicht ben jumgen Rebenftåm: men, fie gebt febr fark um fich), unt in eitue faft

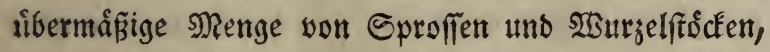
fo Dấ bie Stånme nur febr felten boch uno frark werben, wenn man jene nidht immer bavon abnimmt, da fie benn bis etwa Io Fur boch gegogen merbent 
fónnen, aber bod) immer (chlanf und (d) fwach bleiben.

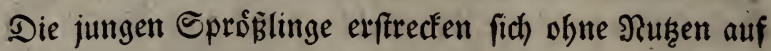

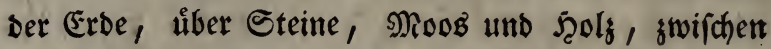
weldyen fie ibre ůberall aus ibren locfern, faftreidjen Sinben beraugłommenbe Saugenurzeln einlablagen; werben fie aber álter unb fefter, fo laffen fie fid) in gerabe und ftånfere Ståmme ziehen. Shr bunfelgrư thes abriges laub ift breiblåttrig, unb fteht einjeln Ins abnedyjelnd auf ipannenlangen, oberwårts breis theiligen, ftarfen Stielen an ben jungen weichen Iries ben, weldje gefdynitten ober abgebrod)en einen zåa)en mildhichten Saft geben. Die Slåtter finb oberwårts glatt, unterwårts aber bei uns mit einem feinen,

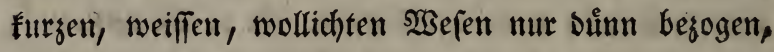
fonft aber, fo lange fie jung fins, mebr roth, tuns fallen im Şetbfte ab. Der ?ans an ihnen ift meits (d) weifig unb frumpf, flach ober tiefer gezacti, zumei: Ien gan shue alle Zncfen. Die augigefdyciften 2 Sin:

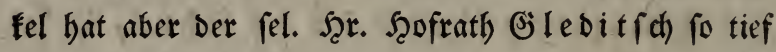
Daran nid)t finben fóntuen, Dấ fich Die Slåtter, wie anbere fagen, mit Dent (sid)enlaube vergleid)en liefren. Die $\$$ flanje treibt nod) im Junit: feime uno buinne, bellgrůne, åftige und fpisigige, fleinblůthige, etwa smei= jollige, eingelne soer gepaarte Silumenftråußer. Da: bei bemerft man månnliche von ben weiblidjen gans abgefonberte Stråudher, woruter bie erften etmas fleitere Sluithen tragen, als bie leştern. Beiberlei

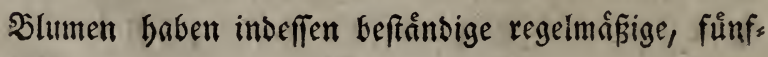


theilige aufrectffftefende Relche, ifre Slumenfronen find fưnfblåttrig uno offen, bie einjelnen \$låtter eys. runb. In ben månnlichen wirb man 5 fehr furge Staubfáben mit febr fleinen Staubgúlb̈chen, gemabr. Dagegen ift in ben gleidfforrmigen weiblid)en $\mathfrak{b l u t t}=$ d)en allegeit ein rumblid)es Fruthtfnetthen, obne Spus

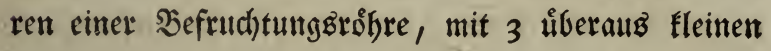
uno zarten herfforrmigen ఏefruchtumgsinarben. Die Fruichte fint rundliche, afdffarbige, glatte, tief geftris d)elte Rerne. Diefe (h)ơne Zergliederung bes gans zen Gemåd) jes babe id) aแb beb bereits angefübrten

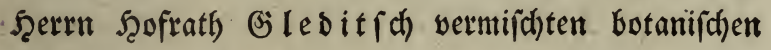
Abbandlungen I. Th. I789. ङ. I62 u. f. gezogen,

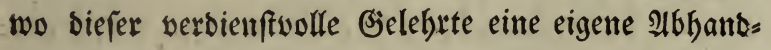
lung ůber biefen Gifftrebenftraud) nittheilte, unb wo: 3u ifm eine Gefhichte bie fich in Eroffen mit felbigen jutrug, weranlajte. Nan pflanste nåmlid) I 769 aus Jrthum biefen Ctrauch als ein (d)lantes sebenges wåd) zut Sefleidung einer. Sonmterlaube ftatt bes

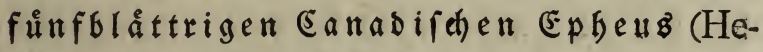
dera quinquefolia L.) weldher nod) befannter un= ter ben Namen bes fo genanten wilben 23 ein fto $\mathbb{C E}_{\mathcal{B}}$ ift, in eimem fleitren Garten, ber mebrere Jabre von ben Eimwohnern eines in ber গåłe bes

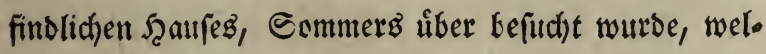
d)e aber auch hauptfädlich um biefe 3eit, wo bie Alusbrimftung bes (jemád) jes am lebbafteften wax, eis ne gewiffe Rernflfeit, mebr ober weniger, augsuftes शै 4 het 
ben hatten. Sie befamen nåmlid) ein Jucfen ber Şautt mit fdomerłbaften Empfinoungen begleitet, wo= rauf Slafen, Entzůndung ber Şaut und Atuffd)mellen Des Siorpers folgte. -

Şerr $\mathfrak{M}$ ůller, ber Şårtner bes afabemifđhen botanifden Giartens zu Serlin, pflangte biefen Saum fut einer anbern Beit um, unb weil er burch bie $2 \mathfrak{t}=$ beit in Edhueif gefommen, grif er ins Eefid)t uns wifchte fich ben Sdhmeißs ab, ba er zuvor siejen Baum altgegriffen uno wahricheinlich etwab von peittem $\bigodot_{a f}$ te an ben Scainden hatte; bies brad)te ifm eine febr beftige Stranffeit zumege, bie fein Seben in Gefabr

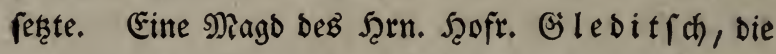

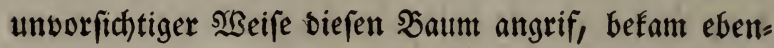

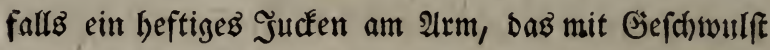

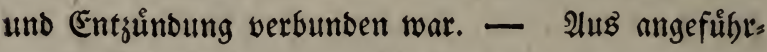

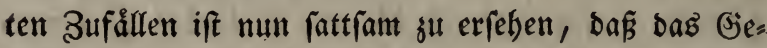
wåchs, von meldhem bier bie łebe war, fefr giftig

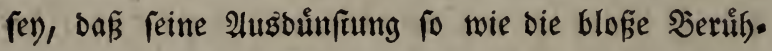
rung beffelben foabliche SSirfungen bervorbringe, uno ber Saft, wenn er bie Şaut beruigut, als ein febr freffendes und nachtheiliges sift wirke.

Im 5 fen uno 6ten Stůcf ber d)emifhen 21nna:

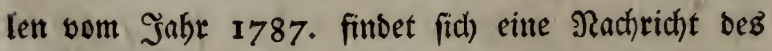
f̧ren. Director $\mathfrak{A}$ d) a bie berfelbe ůter biefen Gifftbaum angeftellt batte, um 
peine Seftanbtheile ju fennen, unb bie Art unb S্sei= re, wie rein Gift allf werffhiebene Thiere wirft, jut beftimmen.

Der wurgelnbe $S$ umady, ober ber flei ne Esiftrebenfteaud) (Rhus radicans L.) bat mit ber vorhergebenden 2 rt fébr vieles liberein, "mb eB fonnte nod) nidht ausgemacht werben, ob er nidht eine blofie Albănderung Derfelben fê. Die jungen, weichen Reben biefes Getwädhfez, winben fidh um bie

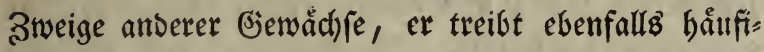
ge Saugemurgeln, Durch bie er fich ůberall befeftis gen fann, und in feinem Waterlanbe Canada, Nir: ginien uno \$enfilvamien, fteigt $\mathfrak{e r}$ bis in bie Cpitze Der Såme alf, er wåd) ft aber aud im freien gera= be auf, boch in niebrigen (d)wachen Stâmmen. Die Slâtter fint etwab fleitter, als bie vorigen, glatt und ofne Zacfen, unt ficlen aus bem Grinen mehr in bas Sothe, Das junge faub aber ift fefr braumroth, bie Şluthen fino leichtgruin, und bie Frucht trocken, und won eben ber Farbe. Der Gaft, Der aus Der Sins De bez jungen Şolgez tritt, ift braungelblich. Das Sekuீhren unb bie 2lusbuำfung erregt, fo wie bie vors bergehende gruffere Ant, alle bie tiblen Bufălle mit eben ber f̧eftigfeit. (S)lebitfd) am angegeigten Srte. 
(sines ber vorgůglid)ften Gewådjê, weld)es peis ner befonbern (sigenifhaften wegen, bier nod) ange:

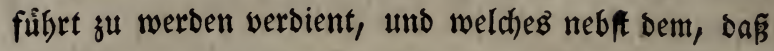
es bejonders in grop̧er Ģabe eime fhnell to̊denbe Siraft bejitet, gleid)wohl aud), wenn es mit $\mathfrak{B o r f i d j t}$

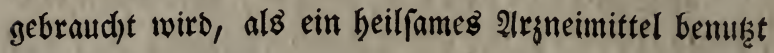
werben fann, ift

Der Rirfdolorbeerbaum (Prunus Laurocerafus L.) Diejer \$aum måd)f nabe ant fdnar: jen Meere wilb, und wurbe I576. von Trapezunt nad) (Europa gcbrad)t. In Franfreich unb Englanb

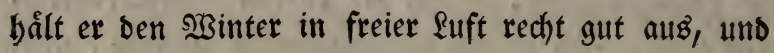
man pflegt ihn bafelbft in Suftwålder, bie er aud)

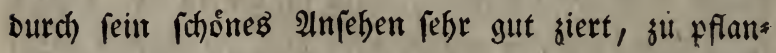

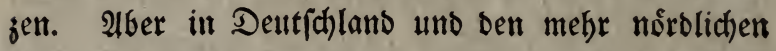

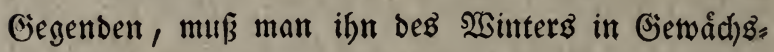
bèufern aufbemabren, uno bennod) blübet er felten it biejen Giegenden. Seine Dumfelgrumen perenniren= ben Slatter futb bicf, eyrunb långlid), ant Rande

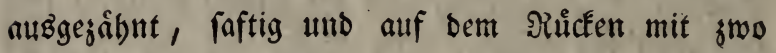
Drufien verféten. Die \$llumen unachen traubenför= imige buifdhel. Die Fruid)te find (c)wairglid), fleifhig, runo, mit einer Epitze uno Sern verfeben.

Sowohl bie Şlumen als blatâter fino wohlries d)end, uns idmedfen wie bittere Mandeln ober Pfirs fichferne. Wan pflegt in gemeinen Reben offters bie ふlåt $=$ 
Slåtter in heiffe Milch gu werfen, um ihr ben anges fîhrten Nanbelgefd)mack mitsutbeilen, uno ben Thee, Raffee uno andre Speifen, benen man Mild binzll: zulekęen pflegt, angenehm idhmedfender zu mad)en. Die: fer (sebraud) ift aber nid)t ber befte für bie Siefunb: beit, weil man in (Erfahrung brad)te, baß̃ biefe Slåt: ter ein Gift befişen, bas wenig andern Giften, in Anjehung feiner Sceftigfeit, meicht. - beponbers ift bas vou ben Slåttern abgezogetwe Waffer von ber

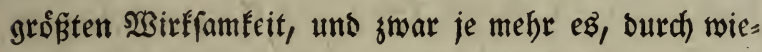
berbolte Deftillation ůber neue $\mathfrak{b l a ̊ t t e r , ~ m i t ~ b e n ~ o ̊ f l i d ) = ~}$ ten und wirfanten Theilen berjelben gefdhångert wirb, won weldhen es audh ein milchichtes 2 njépen er: bålt, uno in welcher aufs ho̊chffe verftårften Geftalt

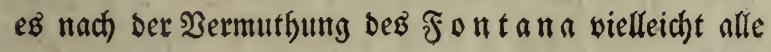
anbre Gifte ůbertreffell warbe.

Der গมf Saft berfelben, ift swar aud) giftig, aber bei weiten nicht fo fefhr, wie bas aus ben blättern beffillirte W̧affer. Diefés to̊otet augenblidflid) alle Ibiere, fie mógen ein faltes oder warmes $\mathfrak{b l u t ~ b a b e n , ~ w e n n ~ e s ~}$ ifnen in irgenb ciner beträd)tlichen Menge burdh ben

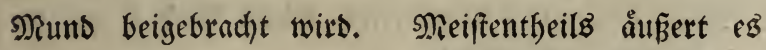

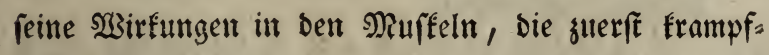
bafte Serwegungen erleiben, unb barauf paralitifd erichlafft werben. Es fa)eint blos wie ein నerven. gift gul wirfen; benn ein foffel voll, ber einen grofen 
Şunb têbtete, batte feine (Entsưnoung verurfacht $\left.{ }^{y}\right)$. X্senn bies rifchen Ro̊rper applizint wirb, fo verurfacht es gleid): falls Ronvulfionen und ben TOD: aber benumberns.

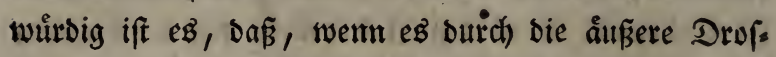
felaber ber Slutmaffe beigemijh)t wirb, es wenig ober gar feine $\mathfrak{2}$ Sirtutigen berworbringt ${ }^{2}$ ).

Der beruid)tigte ald)emift, D. Price in Lonbon, bract) fich in 2luguft 1783 att Dergmeiflung, um Das כetruigliche feiner vorgegebenen Sunft crfannt ju! fécen, mit biejem șaffer ums leben.

Inter alfen biş ieţo befannten Gegengiften ift fette Millh bas juberlåpigfte, titto biefe mag wohl

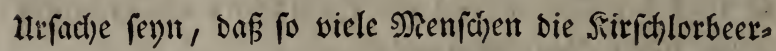

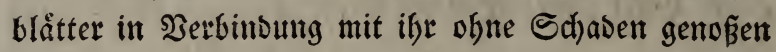
baben. - Nan traue biejer Derniffung aber ia

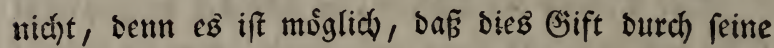
Nenge ber Slåtter, ober cine nicjt alfju fette Nild uns

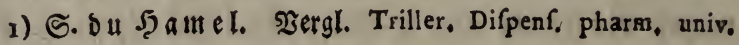
P. I. p3g. 118.

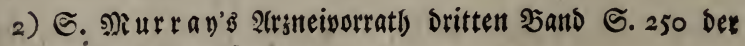
Deutichen tteberfekzung.

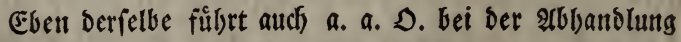

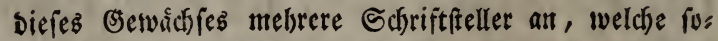
wobl in England als Sranfreich Die widhtigften unt relts famfen Erfabrumgen uno \$iseruche nit Diefem 2 affer gemadit uno beforieben baben. 
uno jufâflige Difpofition bez Ro̊rpers, gleid)wobl auf biefe Art (d)ådlid) werben fam, wovon 2 ater ${ }^{3}$ ) eime trannenbe (jefdidd) ergåglt.

SSleid)wie aber bie Natur fich mefrmals bemủht bat, in viele ifrer fonft idhådichen \$rodufte gleidf. mobl aud) nebenbei eine beilfame und für bie menfd).

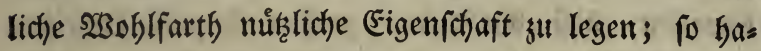
bent audh ebenfalls weife Aergte bas Siridhlorbeers

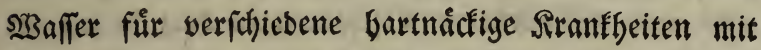
ఇuken ğ gebrauchen gefunden.

Ein \$fund 2 affer von einem \$funve Slåttex abs Seftillirt, iff, wenn es in geforriger Dofis mit 2oors ficht gegeben wirb, ein wirffames Diuretifum, uns

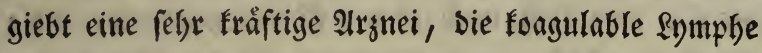

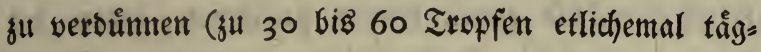

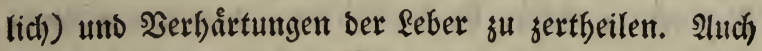
Der: 2ufguf ber Slåtter hat fich åuß̉erlich) uno inner. lid) als ein fråftiges 2(uflófungsmittel erwiefen 4). Iber in grof̧er Gabe bewirft ez allemal Sdjwindel, Angft, Ronoulfionen, făbmung und ben Soo.

Det

3) Diff, de Laurocerafi indole venenata. Vitenb, 1737, p. 18.

4) S. STabnenann und yan ben Sánde Tennsei:

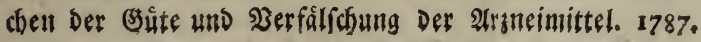
ธ. 102. 


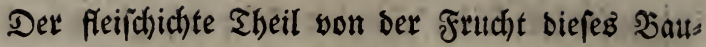
mes ift unfchåolich, unb wirb von 2 o̊geln gerne ge: freffen, audh verbinbern bie giftigen (Eigenfdaften bes Baumes nidjt, Dấ feine ben Sonnenftrablen ausge:

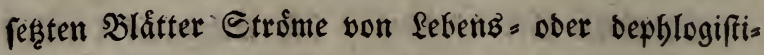
cirter \&uft ausgauchen fouten 5). Diefe eben be.

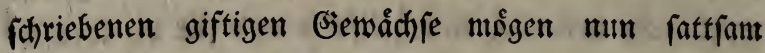

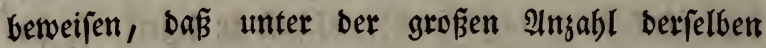
fid) vielleicht nod) mandse vorfinten moigen, welche unz noch auffallendere Ericheinumgen barzubieten im Stande find, befonders da uns bie meiften bes

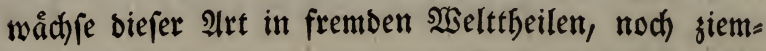

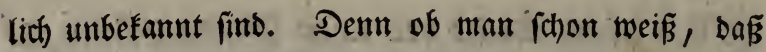
biefe Gegenden, und infondergeit bas fübliche 2 merí: $\mathfrak{f a}$, felye reid) an bergleiden Produften fev, to bat eb bis ieg̨o boch nod immer fdwer gefjalten, viele siefer (ङemåd) je genau fennen zu lernen, indem sie ఇieijenben, weldhe fie befhrieben, uns mur mehren= theils inbianifhe ober andre unbefanute ?amen von ibnen $\mathfrak{a}$ berliefert baben.

Det beruifmte unb verbienftuolle Naturforlcher,

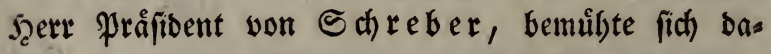
bero etwas ลuverlápigeb von einigen 2 merifanifchen điftpflanzen ju erfabren, unb liefert im 19. Etrict

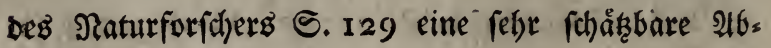

banb:

5) Ingenhoufz Experiences fur les regetaux, p. 233 . 
banblung ůber bas Pfeilgift ber Amerifanter in Guias na unb bie Gewåchje, aus benen $\mathfrak{E B}$ bereitet wirb. Der lefer finbet in Derfelben nidht allein feltne $\mathfrak{b} e$ merfungen ůber mebrere Pflanzengifte, beren fid bie

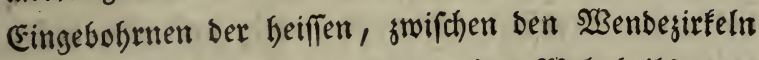

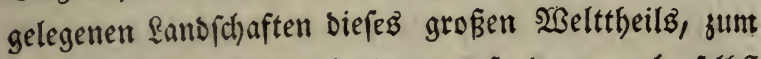
Sergiften ber Pfeile bebienen, fondern auth felbft einige Seftimmung Der Gerwåd) fe, bie zu biefer 26 . ficht gebraudit werben, unb weld)e ber Scerr \$råft: bent nach betren von einem Freund aนร Surinam ifm mitgetheilten aneigen, und anbern eingelnen Theilen Derfelben, aber freilich wegen ibrer unvollfommenteit nicht gang gu feiner Sefriebigung, muீhfam unter= fluchte. Indeffen batte er bodh bas 2ergnuigen für feine Semůhung unter biefen Pflangen eine neue. Pfefferart zu entbecfen, bie er unter ben Namen PIPER geniculatum auffellt, aber Eeinen Gift in berfelben vermutbet. Er erfielt biefe Pfange unter ben fremben গamen Sisoreacobbacoura ober eis

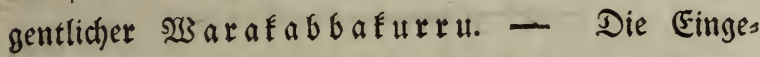
bobrnen nennen biez Gewåd) barum fo, weil eb an ben Etengeln Rnoten hat, bie fie mit ben Rniefno: d)eln bes Trompeteruogels (Pfophia crepitans L.) vergleichen. Diefer Dogel heif̈t auf arramaffifd $\mathfrak{S} a \mathfrak{r a f} \mathfrak{a} \mathfrak{b} \mathfrak{b} a$, unb $\mathfrak{H} \mathfrak{f} \mathfrak{r} \mathfrak{r}$ bas Sinie. Die vout

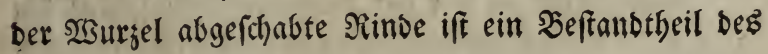
arrawaftifchen \$Peilgiftes. 
Alle bishbero autgefibrten Pflangengifte werbet aber unftreitig von jenem Indifhen Oift ûbertroffen,

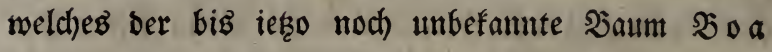
$\mathfrak{U} \mathfrak{a} \mathfrak{B}$ liefert, wobei man aber, um ez ju erbalten,

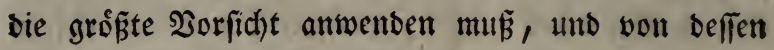
Sråften uno Gebraud) uns fowohl mandherlei wahres, theils aber auch viel wibernaturliches und unglaubs liches erjåblt worden ift.

Da id) nun ber Ģuำ eitte zwei Differtationen uber biefen Gegenftand ju verban= fen batte, wovon bie cine won Scern $\mathfrak{A}$ ejmel $\mathfrak{a}$ แ

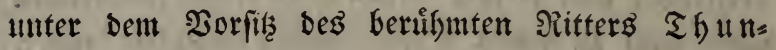
berg gu lufal gebalten worden ${ }^{6}$ ) unb bie andre von Şerrn $\mathfrak{S} \mathfrak{u}$ d) of mit einer ausgemahlten Rupfertafel in paris eridjienen ift ${ }^{7}$ ), fo befinbe id) midy in ber 2erfaffung, sen Şauptinfalt Derfelben bier zu be= musen, uno jugleidh aud) anbre Э?achrichten, bie id) bieruber gefanmelt, mit beigubringen.

6) Christ. aejmelati differtat. de Arb. Toxicar. Macaffar. Upfal. d XXI. May MDCCLXXXVIII.

7) Differtat. fur L'ipo, efpece de poifon fubtil, dont fe fervent les fauvages pour empoifonner leurs fleches pa: $M$. Buchoz à Paris.

\{tud) nel)ne idf bier nit Tergntugen Gelegenbeit, bent

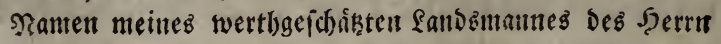
Dr. $\mathfrak{P}$ íf it mit anjufílbren, Der in feiner mater. venenar. regn. vegetab. Lipf. 1785. P.177. von Diefem ङifte eben: falls Neldoung thut. 


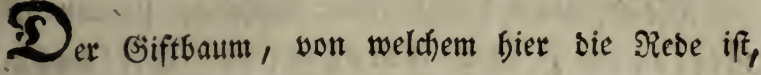
bat fid) biz ief̧t ber Unterfitchung ber Sotanifer entzo= gen ; ร̧errn 2 ejmel åu Differtation inbeffen mabricheinlid, baf er mit bem Ceftro und befen Dermandten unter eimerlei natuir= lichen Sronung ftehe, wenn er nicht gar zu ber Gat= tung bes (Eeftri gebơre ${ }^{8}$ ). (Es wåchfi biefer Saum in verfchiebenen heiffern Begenden Sftindiens, befon=

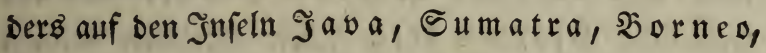
Saleija unb Macaflar bei Maras neben 5 u. bijon, unb befonderz auf Eelebes bei Tora Dios. Er liebt mufte Derter und nackte ミerge, und ift aus ber Ferne leicht ju erfennen, weil in ber গåábe Feine anbern Såume ftehen, unb bie Frbe um inn berum unfruchtbar uno gleich) jam verbrannt ift.

Die

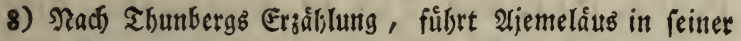
Differtation §. II, Not, I. an, giebt es auf Dem $\mathfrak{Y u r g e s}$ bưrg Der guten Sogfrnung ein felbr gif́tiges Seftrum, (in

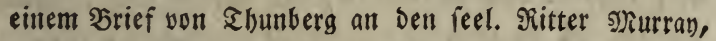

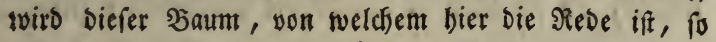

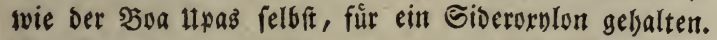
G. Sơttingi(ches Tafchenbud) auf tas Jahr 1788. S. 185 - 187.) Defien Saft Die Spottentotten nit Den @ chlan: gengift vermilchen, uno podann ibre sfeile Damit vergifs ten, weldbe Dadurch noch tods(icher werden, als burch Das

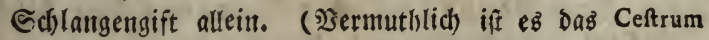
venenatum burmanNi prodr. Florae Capenfis p.5.) 
Die Dftinbien berognenben f̧ollanber nennen ifn ङiftboom, ober macaflarfhe Siftboont einige Spat tenboom. Die Sacaffaren unb Eeles ben nemnen ifn $\Im^{p \nu, ~ u n b ~ b a ~ f i e ~ i f n ~ a l s ~ z w e i ~ v e r: ~}$ fichiedene Såume betrachten, nennen fie ben weiblis

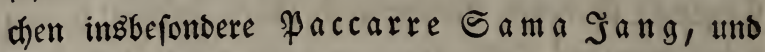

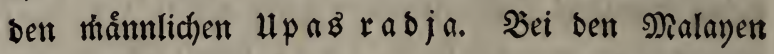

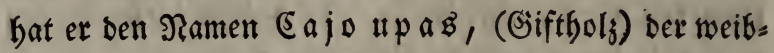
liche aber befonbers Maccan Eavul, unb ber månnlide Diato matti. , Die Malafen nennen

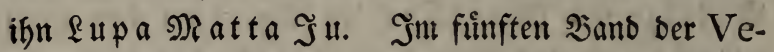
ckofkriften foer Lackare och Naturforfkare

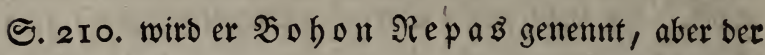
Şr. Profeffor Thumberg glaubt, er múpe rid)tiger $\mathfrak{o} \mathfrak{a}$ u $p$ a s bei ङifft. (ङ. Aejmelaei differtat. §. II. Not. II.)

$\Re \mathfrak{u} \mathfrak{m b}$ nennt ifn in feinen Herb. amb. T. 2 . p. 263. wo er auch Tab. 87. abgebilbet ift, Arbor toxicaria ${ }^{9}$ ). (Es beidireibt Derfelbe jwei $\mathfrak{A n t e n}$, be=

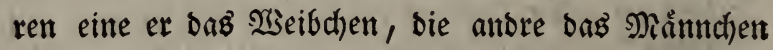

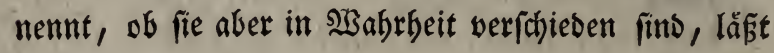

fidi)

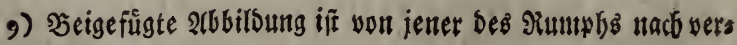
jüngtem 刃iaasftabe Fopirt.

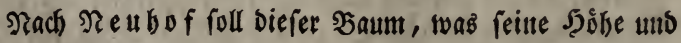

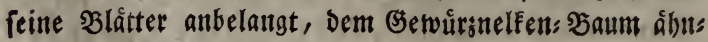
lich fenn. S. Puihn, mater, venen, 1. c. 
fid) aนb feiter furzen befchreibung nicht beftimmen. Diefer Saum bat einen bidhten Stamm uns abftes bense Alefte, eine runglichte, afchraute Rinse, weis: lich)t gelbes, fawars gefleftes unb bichtes Şols. Die Slåtter fino zerftreut, faft ftiellos, långlicht, fpizig, gan's, bie Sippen parallel laufeno, oben rumglid)t, unten wolligt, smei Boll breit, uno eine Spanne lang. Die Frucht, bie man fhon vor vier Jabren bavon gefammelt batte, war aufierorbentlid) trocken uns bart, ounfelbraun, und batte einige 2 ehnlichfeit mit Saalls åpfeln: bie åuffere Echaale war fo febr an ser innern

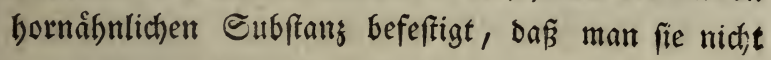
Davon trennen fonnte. Der månnliche Saum foll einen Gơhern Stamm und gebrångten Sopf, fleinere uns weichere Slåtter haben, ůbrigens aber bem weiblid)en vollfommen åfulid) fenn. Die Sbluthen fint unbes faunt ${ }^{10}$ ). Der Saft biefes Saumes, ber eigentlid' 32

sie

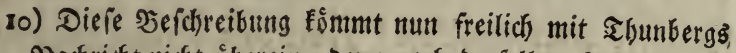
ग2achricht nicft úberein, Detn nach Derfelben fommt unfer

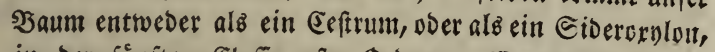
in ber fuinften Elaffe erfie Oronung (Pentandria Mono-

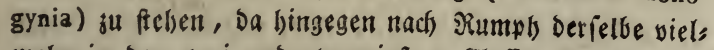
mebr iit Der twei und ztwanzigften Claffe (Dioecia) zu fuchen iff.

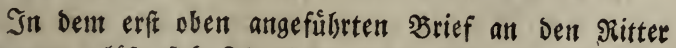
Murray, lábt fić) Tlyunberg unter andern verneljmen:

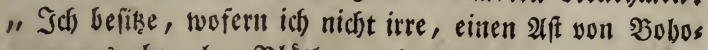

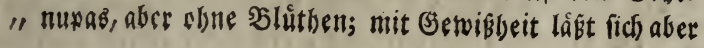


bie tooliche Riraft befist, ift braun f(t)ivals, unb wirs inbem er austroçnet zu einem Şarz, bas bei bem fos genannten Månnchen allezeit bårter unb (đh) wånger iff, unb faft wie \$ech an ber Şiţe Des Feuters zerflieft.

Das Sammeln biepes hargigten Saftes, weldes

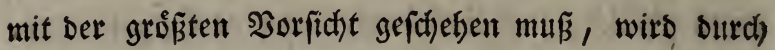
Seute unternommen, melche bie Nacaffaren $\mathfrak{E} e r a$ gi a nennen. Sisenn fie ben Eaft fammlen, fo ges

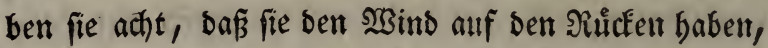
anb foldter nicht von bem Saum fomme, Damit nicht Der, ber ifn fammelt, in Sebensgefabr geratbe: ald

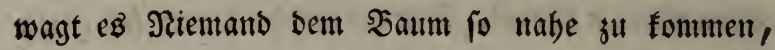

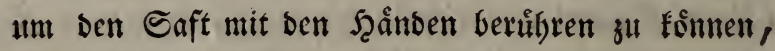
und itan fångt bafero benjelben aljo auf:

Fs werben nåmlich lange Sambusiro̊fke, oben mie ein 23 urfipies gugefpizt, uno nit móglidjfter Giewalt idfief in bie Rinbe Des Ctammes geftổen, Damit an ifnen ber blutrotbe Caft ${ }^{\text {II }}$ ), ber balb hernad) bidt uns baut

" bocb nicbt beftintmell, ob er won eben bem כ̧aum ber " Fev, fo lange noch siemanto das Şer gebabt bat, einen

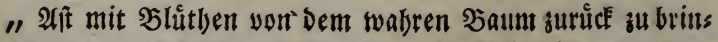
" gen, weldjes gewis meniger gefábrlich) ift, als ber " Gaft voer Das Gummi zu bolen, u. f. w.

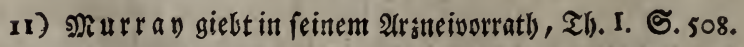

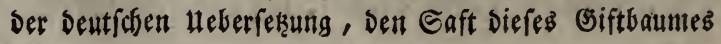
milds: 


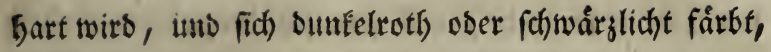
berabtropfe. Je náber an ber $2 \mathfrak{S}$ urzel ber Baum vers

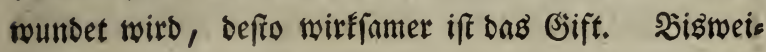
2 3

len

milchartig an, vielleicht if Diefes aber ber Saft bez blinbs machenden গ̧aumeż, Excoecaria Agallocha L. won welchem

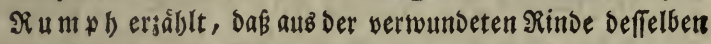
mit Setwalt ein icharfer milchidfter Saft berauşringe, Der, wenn er in bie 2ugen fprise, grobe Ecfmersen uno bei lange zogernoer Saúlfe Sflinbbeit verurfache, wie fols d)es bie bollàndirchen झiatrofen bei ibren erften offindis

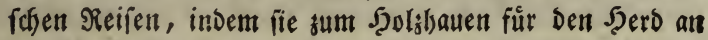

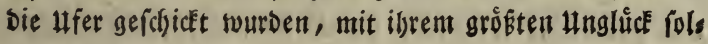
len erfaberen baben.

Gollte nicfe auch Der ஒaum bier mit in \$sergleidf su . ftelsen fonmen, weldben $\mathfrak{P a t}$ ter ron am angeseigten Drte S. 169. unter feinen vier befirtiebenen afrifanis nifhen Pfansengiften, als eine Ant Gerberbaum (Rhus) Den matt alt großen oder Dranien: Slufe fittbe, ans fúbrt? unto von welchem er fagt, Daß̧, tween man Das sift

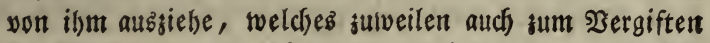
Der $\mathfrak{P}$ feile gebraucht wurdoe, mant fich Die 2utgen bebeckent múffe, benn der Eleinfte Tropfen Der fie nur berúbrte, múrde fie ber Gelferaft berauben.

Eljebem glaubte man, won biejem blinbmachenden Baum fáme bas feines 2 obllgerudfis ivegen berúlsmte, und in

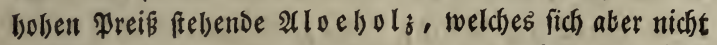
befiáttigt, ba man ieķo Den Saum, von welchem bas Şot Esmmt, Durch Den Stin. won \&our e ir o, einen Portugies fent, in beffen Flora Cochinchinenfis weldfe 1790. in 2 bănts Den su Siffabon erf́chien, genauer fennen lernte. Er if

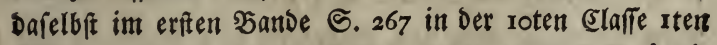
Dronung als ein eigenes Ssenus, unter ALoExyLum Agallachum alfgenommen worben. 
Ien werben mebrere, bis zwangig bergleidhen roofre an bem Saum angebracht, bie man brei bis vier Tage Daran Iáft, Damit ber Eaft in ifren Scêblungen fich fammle und anmadje. Dab vberfte Slieb einer fol= d)en $\Re \mathfrak{d} h r e$ füllt fich bann mit Saft an, ber ůbrige Sheil wirb abgefduntten. Der nod) frifdee Saft in

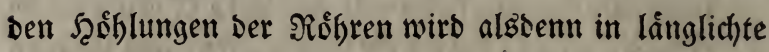
Stůctchen ober Sịgelchen geformt, unb, mit Reinmano oft zehnmal ummidfelt, an einem temperirten Sort aufgehoben, pobann múffen aber biefe Rügelchen gleid)=

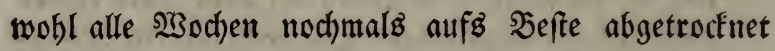
unt gereinigt werden, bamit fie nicht idjimmlicht wer:

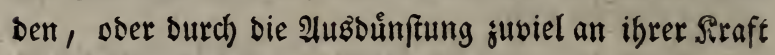
verliebren.

Die Rraft bes Giftes biefes Saumes ift aufferor:

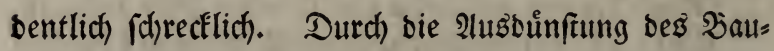
mes empfindet man fogleid) ein heftiges Jufen ober Růtzeln an allen Giliebern, bie balb barauff fteif werben, alles Eefühl verliebren, unb wie criroren, ober vom Sirampfe befallen zu feyn (cheinen. Mian butet fich Dabero biefem \$aum ĭ nabe fll fommen, obne juvor bie 21rme, Fůpe, Sd)ultern uno ben Sopf mobl mit Sleibern zu bebecfen. Derjud)t es jemano mit entblógtem Şaupte unter bem Baum fult ftehen, fo gehen ifm bie Şaare alts, uns fallen einige $\mathfrak{T} r o=$

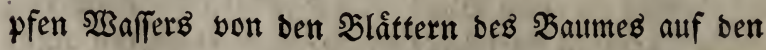
leib, to fabrillt ber betroffene Sheil fefre auf, wie 


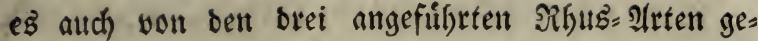
rdjiegt. Nian finbet um ben \$aum herum auf ber Erbe gewoshnlid) Soogelfedern, unb biefe moigen berweis

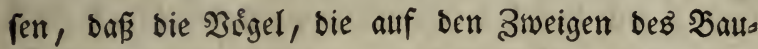
mes fich nieberieţen, todt zur (srbe fallen : úbrigens

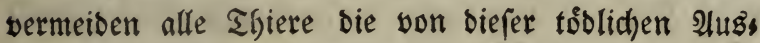
binftung geidhwångerte $\mathfrak{B u f t}$, und $\mathfrak{e B}$ ift, als wenn in

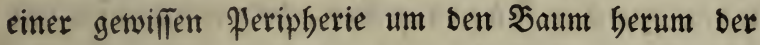

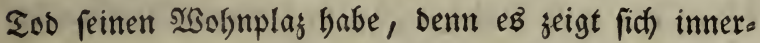

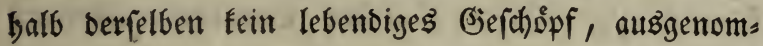
men auf Selebes eine gefởnte Eddlange, (Serpent

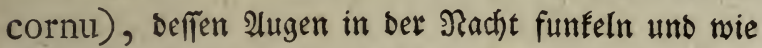
Gener lenditen follen, uno welche sie Macaffaren Illar bal u nemten ${ }^{12}$ ). Wuch wirb firt wabr aubs gegeben, baß̃ unter bem baume formobl, als auth foo gar einen Flintenffur ober Steintwurf weit won Dems felben, Fein \$fhånjohen, Staube, doer Giras machie, und die (Eroe ganj unfuctitbar fey.

$$
\text { 2. } 4
$$

$\Re \mathfrak{m p \mathfrak { h }}$

12) Sermutflich ift es bie Coluber Ceraftes Linn. Sie ift áfferff giftig, von grauer Farbe, und I8 bis 26 . Soll

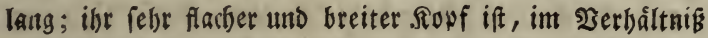

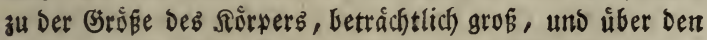
2urgen erleben fidi kleine Sefjuppen, weldhe Shorner ges

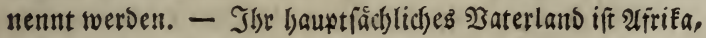

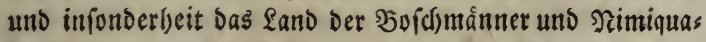
Sottentotten, Die Das Gifit Derfelben zium \$ergiften ibrer

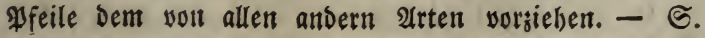

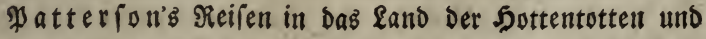

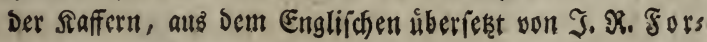
fiter 1790. S. I58. u. f. 


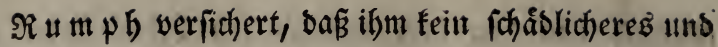
(d)recflicheres Produft im Pflanjenreiche vorgefommen (fi), als diefes తift, morauf felfft die Snbianer grof thun, und daf bie Gollandifhen Goldaten es fonft

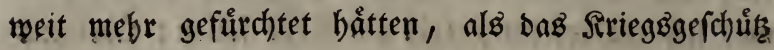

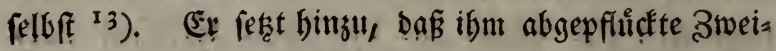
ge an einem grofen \$ambugrobr jugefdicft worben, bie fo wirffam genefen wårẹt, Daß̧ menn man mur

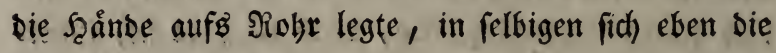
(Smpfinbung åufferte, die Daš Epwårmen erfrorner Gilieber berworbringt.

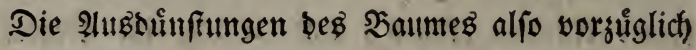
und ber frifhe Eaft, befitenen bie meifte Giftfraft, bie aber bei bem Saft nad) unb nach immer (d)wåd)er mirb, uno fich endlich gang verliert. Das Gift bes.

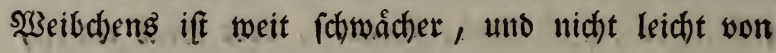
freien Stůçen tóbtlich, wešnegen man eg ạuch jum fangen der wilden Thiere ju gebraudhen pflegt, nidjt aber

13) Dieje Bemerfung baben mefrere Reifébeichreiber an: gefúbrt. SJevdt in reinem Schauplak von iafrifa und

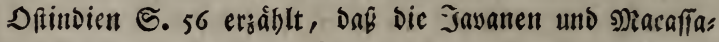
ren fich) einter 2(rt Э̧lašribbre bebientell, aus welchen fie Pieile bliefen, Deren Spizen fie suvor glúend madjten, uno bernach mit einem Ssift beidnierten, welche, went fie abgejchofien twírben und tráffen, abbráchen uno in bet

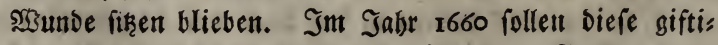

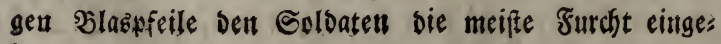
iagt baben. 
aber um bie Pfeile im Rriege bamit ju vergiften, auffer eg̉ nirb mit bem måunlichen, ober einem ans Dern ftartern vermifht. Die vergifteten Pfeile bes balten zwei Jabre ihre Rraffte, boch follen fie auch bistweilen in einem Beitraum won einem ober wenigen Mionaten biejelfen mieber verlieren. Mit biejem Sift fann. man aud), nenn man flug gu Werfe gebt, ob) ne Schaben umgehen, mur barf es nicht ummittelbar in bas \$lut fommen, ja bie Einwohner ber Jutel Eele=

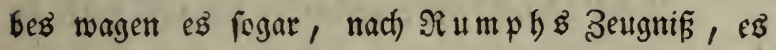
ftatt eines innerlidjen Nittel' ju gebraudjen ${ }^{14}$ ).

Die Rrafte biefes Giftes werben burdh ein gewifs fes fidheres (xperiment erforicht: man frést nảmlich Daz fogenaunte $\mathfrak{R} a m p$ ujang oder Amomum Zerumbet $\mathrm{L}$. ( wilber Ingmer) bis $\mathfrak{e B}$ ichleimigt wirt, bernach wirb ber Saft auggeprefit, unb Durch ein lei= nen audh gefeift: zu biefem Saft wirb algbann et= waß Gift nit ein wenig $2 \mathfrak{3 a f f e r ~ g e g o f f e n . ~ 2 t u s ~ b e m ~}$ (4) nellen 2lufwallen, bas nach siefer Mifhung ent= fteht, und aus ber heftigen benegung beffelben beur: theilen bie Sachwerfånoigen bie Sirâfte. Diefe తెå)= rung wirb oft fo heftig, Dafi in Der Mitte bes Gee=

$$
\text { \5 miches }
$$

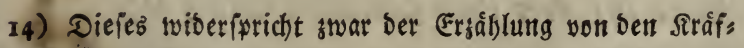
ten biefes siftes, Die uns in Veckof kriften foer Lackare och Naturforfkare finften $\mathfrak{b a n d}$ S. 226 mitgetbeilt ift, Da aber biefe Ersáblung das Begentbeil aller anbern entbált, fo Darf man lie fur siveifellyaft anieber. 
mifches fich cine grofe Girube bilbet, uns man bent כoden bez Gefáąez feben fant. Diefe Speration

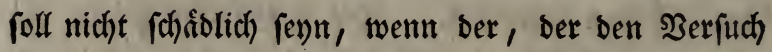
madht, fich in acht nimmt, benn verbid)tet befist uiber. baupt bas Gift burch feine Ausbunftung feine fo befo tigen Sirafte, wenn $e^{B}$ mur nicht ins Slut fommt, unb surd) ben Saft won Zerumbet zut verftánften Wirfung getracht wirb.

Diefes tódliche Gift verbreitet fich und wirft in Dent verwutubeten Sio̊rper Dergeftallt, baß̃ eş heftige

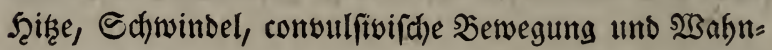
finn erregt, wornuf in furger Zeit, wenn nid)t ichleu= nige 'Scullfe geleiftet wirb, ber Tod folgt. 2An ben Iispern ber Verftorbenen bemerft man bann Petechien

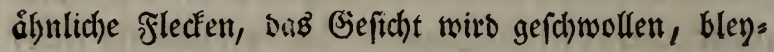
färbig unb bas 2 seiffe in ben 2ugen gelb.

Die ntacaf̣arichen Ro̊nige ftellten mehrere $2 e r$ fuche bamit an folchen Perionen an, bie zum Sobe verurtheilt waren, um zu erfabren, ob eine ßettung vom Sobe mo̊glich wåre, aber vergebens. Wan ver: wunbete mit Pfeilen, sie mit foldhem Gift beftrichen waren, einen Daumen ober eine 3ehe eineb folchen \$erurtheilten, uno (d)nitt rogleid) bas angeftectete Slied $a b$, aber ofne gludflidjen (Evfolg ${ }^{15}$ ).

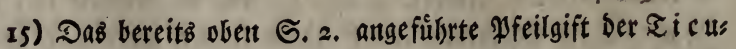
nas und Def úbrigen Nationen auf ভuiamao weldges zu 
Der 2erfaffer einer mit vielen Fabelit vermeng= ten গachrid)t von einem Giftbaum ahf Java, won welchem weiter unten mefr worfome

ment

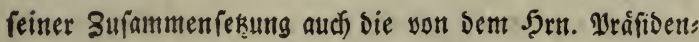
ten von $S$ chreber angefúbrte Wieffecart entbailt, if

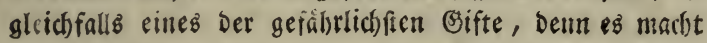
in Der fleinfien Quantitát, wern es Durch eine Sisube

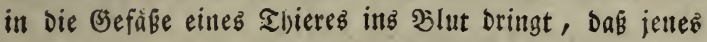
in weniger als einer Jinute, Dem 2nj(chein nach) sbne groke Edhmerzen frirbt, fo Daf bie febengigeifter glcicis: fant unmertlich verlofichen; wiewobl man in bem augen: blicke, Da es ftirbt, bigneilen ichwacise Sonvulionen bemerft.

Wुie feftrer if es aber nicht ebenfalls, bie ttrfache ber

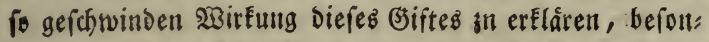
Ders wenu maa bedentet, Doß nur eine io fleme Qun: titát beffelben it einer oft unbedeutenten 23 unde einen to fichnellen ₹od bewirfen fam? - 29 egen Diefer unbes greiffichen (Ericheimung follte man wirflich mit Şrn. D. Soriffant (S. im 17. 2̌. Der Philofophical Transactions) vermutlen, Dá an Der fo fofnellen fes, und Des sbigen Siftes, eine plísliche sufammenties

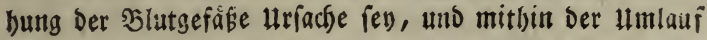
Des ஒ̉lutes auf einmal gebenmet weroe. Diefes fdeint fich auch Daburch zu befíattigen, weil man abgezesbrte Thiere, ober vielmebr folche, bie wenig \$blut melor bat: ten, langfamer an biejem sifte fierben fabe. Der Stes

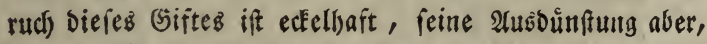
fo wie die 2applifation bes Bsiftes auf Der unverleşteit

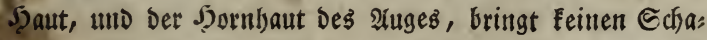

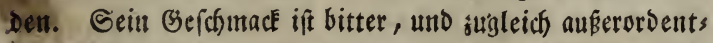
lick. Grentend soer beiffent, sumal wenn es die Atrawak: Fentroch mit rothen $\mathfrak{Y}$ feffer, Capficum, veríkęen, Doch fanu naan es obne (jefabr Eoften, nur Darf es nicht nach 50 on: 
men wirb, will unter anbern verfichern, baß̃ auch Ouellen won siejem Gsift angeftecft und toblid) wer:

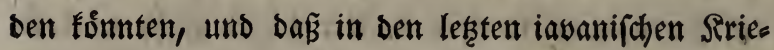

gett

tana in grofer Dofis in einen lecren Meagen gelangen, obne in wenig Minuten tobliche Folgen nach fich su ziefren.

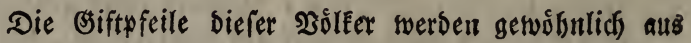
Slastoblen geblafen: fie fint gemeiniglich swolf Boll lang, etwas bicker als cine ftarfe Stricknadel, und werben aus Splittern won Der barten, Dichten, aubern Subftans bes Cof aritobaumes (Bactris major JACQ. amer. p. 280.

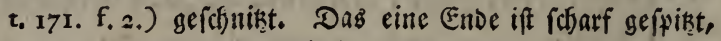
unto wiro mit bem Pfeilgifte beftrichen, um Das more Enve aber wirb eine Rolle baumtwolle gebunden, Die nach

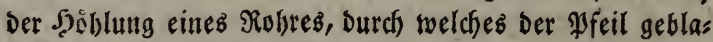
fen wiro, genau eitgerichtet ift. Serderben subereitet ift, fo wiro er in baz etliche SuB lange Siobr gefteckt, uto mit einem einsigen Saaucb Durch Die Szóblumg Des Robres nach Dem ä erlegenden Begens ftand berausgetrieben. Er fliegt fodann ntit groferer Schners ligfeit, und oblue su feblen bis auf eine Diftans von breif fig ober viersig Siutljen, wo er bem Tliere weldbes er bis zun §luten verwundet, eituen fofmellen und unvermeidlis

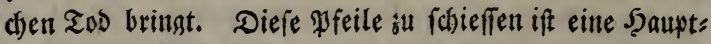
becháftigung Der $\mathfrak{3}$ silden von. Sinbbeit an. Durch lange Hebung erwerben fie fich) eitte faft unglaublicfe Sertigkeit

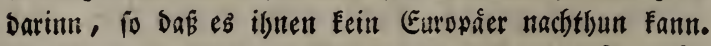
Diefeß zujammenge

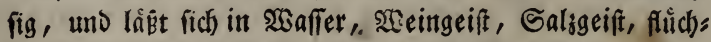
tigen alfalifchen SEeift, Şlut uno Speichel aufiofen. 

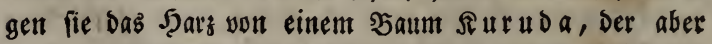
nur an ber Seefannte wácbit, noch Darunter. Suir ein

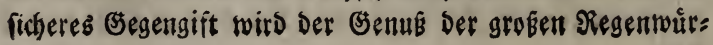


gen bie frollånber bestwegen lebenbige Fif he mit fich geführt bătten, um mit felbigen bie Dutllen vor bem Gebraudh ju průfen. Allein bie vorbanbene ges naue und umftåndliche befdreibung bief̧es Rriegg, bie man in \$atavia von bem ehemaligen Gouverneur f̧arting bat, füfrt nicht baz Gieringfte von biefent Umftanbe an. Es mird Dabero rathfam fenn, biefe Nachricht ebenfalls zu begweifeln, bejonbers, ba nod) nidht ausgemad)t ift, ob Der eingebicfte Saft biefez Saumes ein Gummigary (Gummi refina) ien, uns Das şaffer einen Theil aufófen forme, benn forwohl bie mit Dem Giifte angeftellten ふerfuche, als anore

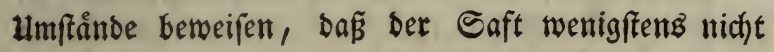
gang

nter uno bes aucferrobrs gebalten: Doch ift in Diejem Fall

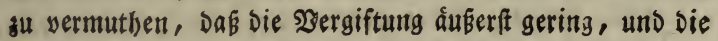

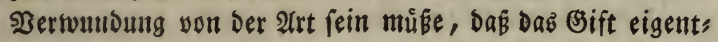
(ich noch nicht in bas \$llut eingebrungen, weil man nach angefúbrter Eigenichaft Deß Gsifteß Eaum seit getwinnen Eann, fich eines ઉsegengiftes zu bebienen. Sar Elbre fưr

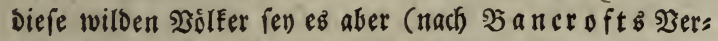
ficherung) gefagt, Daßs fie fich Diefes furchtbaren (siftes niemals gesen menfchen bedienen, fondern fie gebrauchen

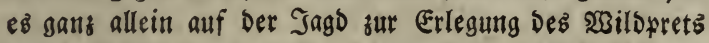
Deffen Sleifd) fie obne Schaben genießen. Die fünf ges woibnlicben Ingrediensielt, woraus Daş amerifanif́che Pfeils gift bereitet wito, und von benen einige melor oder wes

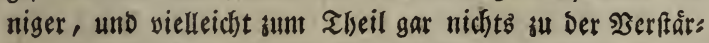

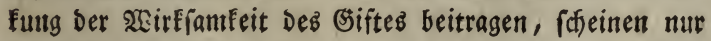

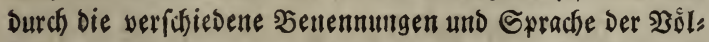
fer von einander unterichieden zu fenn. - Bielleidst if́t Das Gsift Der \&amas in ¥eru von gleicher ஒefchaffenbeit S. von $\Xi$ d) reber stbbaltolung. a. a. D. 
ganj gummiartig (gummofum) fey, weil er vom Strack ( Reisbrantwein) aufgelópt werben fann ${ }^{16}$ ).

Nan

16) In 2 frifa ift ber ङebrauch, 2 Saffer su vergiften, uno wilbe Fliere danit zu erlegen, unter ben Sottentutten allgemein befaunt. Sie bebienen fid) ou biejer 2 bfid)t einer gewiffet

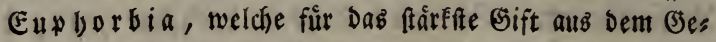
swáchsteiche in Afrifa gebalten, und Defien (summi ebenfalls

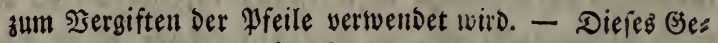

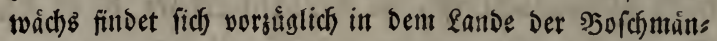

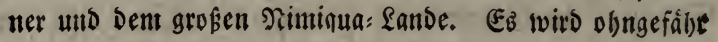

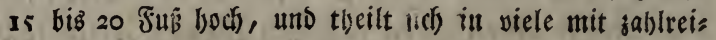
dhen Stachell verílbene aweige. 2 senn Die Eingebolgr:

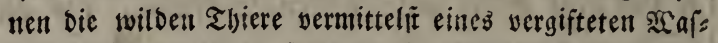
fers. vergiften wollen, fo fiecten fie abgeidhnittene sweige von bentelder Eupborbia in ein von ber Quelle ab: wártż gemachteb loch, in weldjes fie das 2Saffer leiten, bie Quelle aber verbecfen, Damit beat şsild, welchez bies jelbe bejud)t, Eeine andere $23 a b l$ tibrig bleibt, Denn its

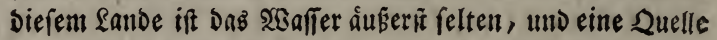
oft 20 刃ieilen yon Der andern entfernt. Scin ミbier, wels. dhes woul cinem folchell 2 safier gettunfen bat, foll bayous

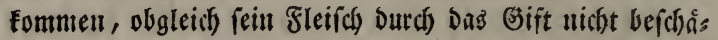
Digt wird. - Patterfon jalse auf biefe art emen ver: gifteten zebra, ser fith noch nicht eine balbe englifche Meile von Dem Niffer entfernt batte, als er fchon binges fallen war. Diefe 2 int Daß 23 affer zu vergiften, vermebrt Die Befalsr fur Sieifende, welche mit biefem 11 mitande nicht befannt fino, obgleich bie Eingebolsencn Die \$ors

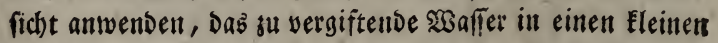

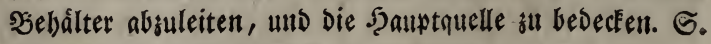
Patterfo ins Peíen. 5.60 und 169.

Sier mocthte freilich einem Teifenden, ehe er trinkt, bet 2erfuch mit lebendigen Sijd)en febr gut zu itatten fom: 


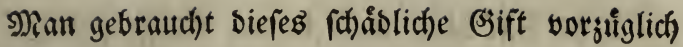
bei verfhiebenen Pationen, um die Pfeile im Sriege bamit zll vergiften, aber was am meiften munberbar ift, fo Gålt man es rob unb unvermifa)t fur ein beils fames (̋egengift innerli(), und auch åufferlich als ein

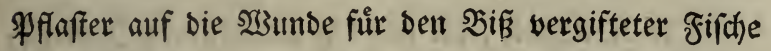

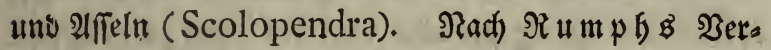
ficherung fifllet es ben Sd)mers gefdwinder und zieht

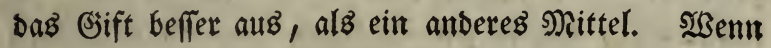
jemand Gejhmire ober Firåtze bat, unb eine \$ille

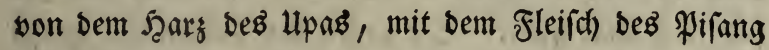
(Mufa.) vermifdt, nimmt, fo foll eb eine trefliche Slutreinigung feun ${ }^{17}$ ). ( (. Rumph. herb. Amb. T. II. P. 269.)

Dณษ์

men, wenn es anbers nur moiglich wáre, auf bergleichers Seifen rolche Gefchipfe lebendig mit berum fübren ju funnen.

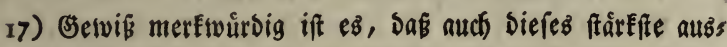

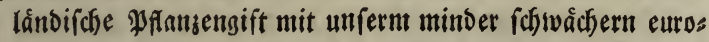

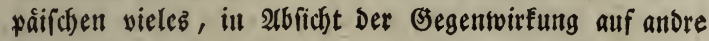

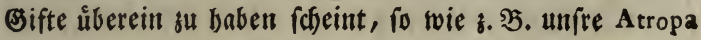

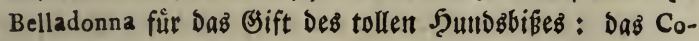
nium maculatum, Aconitum Napellus, unt atore giftige Pflansen nelbe, fứ mancherlei Sranfleiten zum innerlis

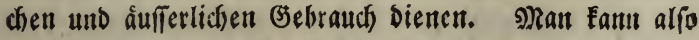

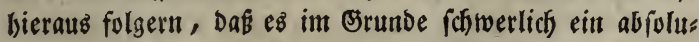

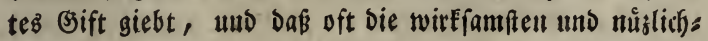
iten Nebicamente, in Récfífift Des Gebrauchs und Der

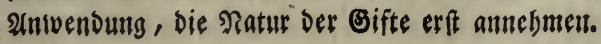




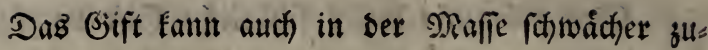

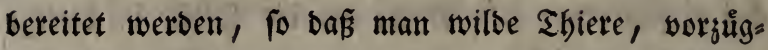

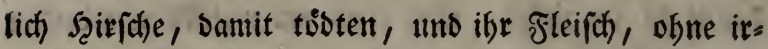
gend eimen Schaden zu befürd)ten, effen fann.

Die Scollånder, bie mit ben (Einwohnern Jnbiens,

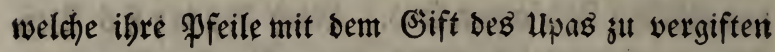
pflegen, Rrieg fůfren, baben verid)iebene Mittel ausgebadjt, wie fie jene tóblichen $\mathfrak{W}_{\mathfrak{B}}$ unben vermeiben fornten, weldhe ntan, wenigftens fonft, gang uno gar für unfeilbar bielt. Eie zogen berobalben fefr grofie und weite Sileiber entweder aus fipanifhem Reber ober aus leinenen Segeltuch an, weldye bie \$lfeile nidjt leidjt Durdjbohrten, und ebe bie Solbaten Den 2ngrif madhten, biengen fie cin mit \$ped) uiberzogenes Segel tuld)

So ebent als idf biefés fobtieb, erbielt idf einen atrs

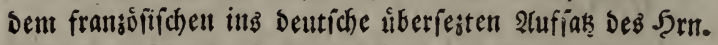
Sitters uon $5 \mathfrak{r}$ ay in Siegensburg, welchen berfelbe der

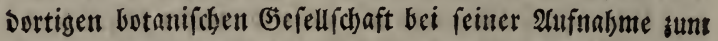
Ebrennitgliede âbergeben bat. - EB if bier ber Ort nicht ansufuibret, mit welcher mablerifchen Schónlseit ber ver:

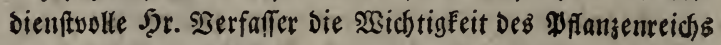
it bemielben fofilbert, uno idf bemerfe babero nur fo viel, baḱ berfelbe unter andern neben ben Echaden, welchen

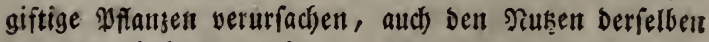
als 2 assmeimittel anfílbrt, fich aber bariunen irret,

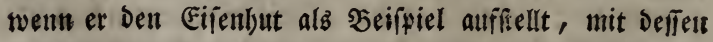
Saft et Den Meacaffaren ibre Pfeile vergiften láffet, uno fonad) jenes gelinoere Pfanjengift mit unfern $\mathfrak{u}$ fas ver: wedjfelt. 
tuch vor, wobutch fie burch eine Definung mit Feues: gewehren ichiefien fonnten, bie Pfeile ber Jnbiamer aber nicht Durchbrangen.

In ben erften amboimefifchen Rriegen nafmen bie Soldaten, weil fie fein anderes Mittel fur biefen. Gift Eannten, ifre 3ufludbt sum - Menfdienfeth, weldhen fie frapen, Darauf erbrad)en fie fid, unb es wurbe bierdurd ben ůbeln Folgen vorgebengt. Die Folge ber 3eit lehrte aber Den Macallaren an= Dere weniger wiberwårtige und edfelfafte Mittel, bie

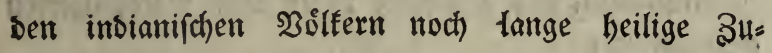
fluchtsimittel bleiben werben, als nåmlid):

1) Der Saft Der gefâuten Zwiebel, ber afia tis fden filiennarzifle, (Crinum Afiaticum L.) verichlucft, und auf bie sisunde ges legt; Daher entfteht ein heftiges (Erbred)en, Dutrch weldjes man bas Gift won fich giebt.

2) Um bie ftarke Şize gu litbern, gief̈t man ben Saft won ber Melonenpfebe (Cucurbita Melopepo L.) auf bie $\mathfrak{S u n d e , ~ u n d ~ i f f t ~ e i n e n ~}$ fleinen Sheil bavon.

3) Die Rinbe ber tra $\mathfrak{u}$ bid ten feige (Ficus racemofa L.) ober anberer Feigen gefăut, ober zerriebel!, unb auf bie $x_{5}$ unto gelegt. Debglei d)en zarte, rothe Fafern, ober zarte Șurzeln 
ciner getwiffen Feige, theldhe bie Jnbianer $\mathfrak{\Im} \mathfrak{a}$ ringa nemnen, theils als einen Trant bereitet, theils aufgelegt.

4) Die Ninde DeB $\mathfrak{A} l \mathfrak{b} i \subseteq a i j a \mathfrak{n}$, vom Etamm abgefhaben unb auf bie șunbe gelegt.

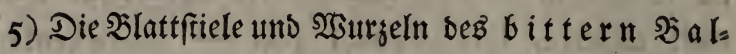
famapfels (Momordica Charantia L.) geftofen uno auf bie sisunde gelegt.

6) Die Sצmzel von Eajo $\Re$ a $j a$.

Linb bann bas gewififefte unter allen macaffarifchen Mitteln ift:

7) Ser mildhidhte Saum, bei ifnen $\mathfrak{P} u l e$ oder Si t e genaunt. Siringt man beffen Slattfticle an bie Sinnde, fo follen fie alles Gift, ja aud fleime Stúcfe ber \$feile, bie aus Meerrobr ge. macht fino, augfaugen.

(Endlid) ift aber unter allen angefůbrten Mitteln

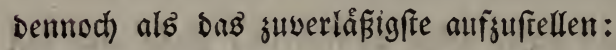

8) Die bittere inbianifhe Schlangen = ober Gifts ww: (Ophiorrhiza Mungos L. ${ }^{18}$ ). Man giebt biefe

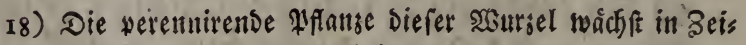

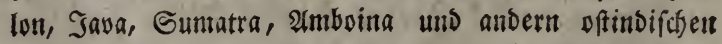
Infeln. Die sesurge? tif cinfuch, wenu fie grob wirs, if fie eime Swanne doer fechs soll lang, eintes Singers bice, frumn, fnvtig, sebogen uno mit einer feft angerwachienen, 


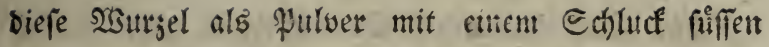
25affers fu einem balben bis gangen Duentchen ein, ober man macht aus bem \$ulver mit Epeichel ober 25affer einen Srey, unb legt ifn auf bie $2 \mathfrak{B} u n b e$.

(5) 2

ง|118

rumblichten, içwaummichten, braunrotben sinbe umges ben, bie einen weiffen, barten, boljigen, zerbrechlichen

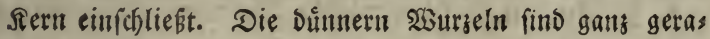
De, Die bickern aber find witÉamer. Sie bat Eeinen Gess ruch, aber cirter erffaunlich, jedod) nicht umangenelynt

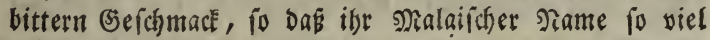

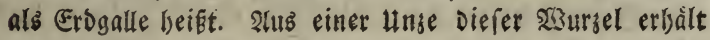

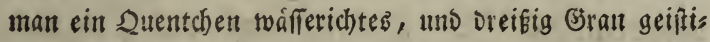
ges febr bitteres Extraft. Siebji Dent, Daß́ man Dieie

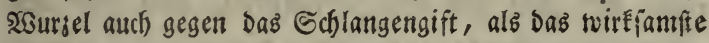
Nittel empfieblt, twird fie aud íberbaupt gegen andere Sifte, und wiber Siranflbeiten, Die ibser Natur nadh jes

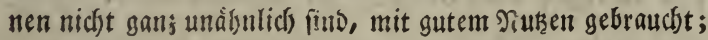

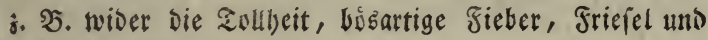

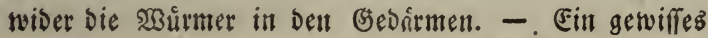
Ctintetbier, Die ミ2 a ng u fie (Viverra Ichneumon L.) wels dhe Die Portugiefen Nango, Die Javanen aber Muntgutia

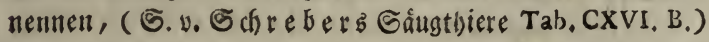
foll, wie man fagt, nachbem fie in Sampfe mit Den

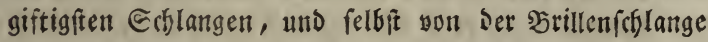
verwumbet worden, ibre Buftudft bu Diefer 2 sursel nels men, und Diefelbe freffen. - Durch fie beilen audb bié Sndianter vollenonmen ifere Sieber, und aus ihrer Şitter: feit Eann man leicht abunebmen, Dá fie cin vortreffiches

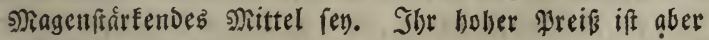

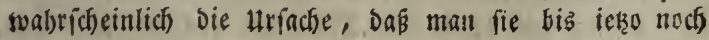
nicht Durchgaingig in allen sapptye Een Deutichlanos einges

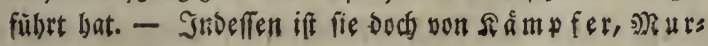


Aนเి fåmtlichen angeführten Mitteln gegeแ sicjes Eifft exhellet aljo, Daf biefelben meiftentbeils fdarf uno fưletend fenn muifien. Ferner muf man Sorge

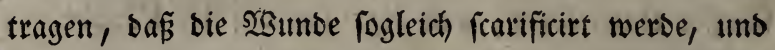
Dẩ man, um aus felbigen bas ङiift ju jiehen, warme

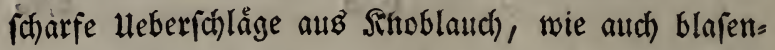
jiehende Dinge und bergleid)en mehr anwende, to sie man interlich $\mathfrak{B r e c h}$ = แmb erweid)enbe Mittel, als shlid)te und (d)leimichte Defofte gu gebrauchen bat. 2lud) muf eine einfadge uno abfinglente Diåt lange Zeit beobachtet werben, weil bas Gift an je= Dem $\mathfrak{2 e r w u n b e t e n ~ n a d ) ~ D e r l a u f ~ e i n e s ~ S a h r e s ~ w i e b e r ~}$ aufleben foll ${ }^{19}$ ). Dabero muß ber Patient ald unter ber Zeit fich bez Zerumbetz, unb aller anbern erfitienden Dinge enthalten, Damit nicht ein SRlut fall erfolge, ber nid)t felten tódtlid) wirb.

\section{Ob}

rav unis ben meiffen Schriftitellern, welche bie Nateria 刃iedica abgehandelt baben, angefúfrt worben. Eine $2 \mathfrak{b}$;

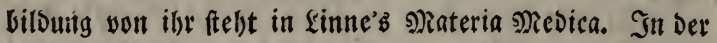
funften $\mathfrak{2}$ uffage biefes aber ibrer Inwollémmenbeit wegen weggelaffen woroen,

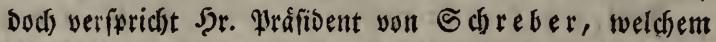

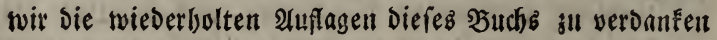

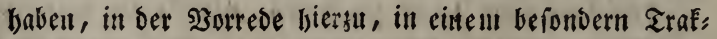
- tat úber biefe Pfanse, Dab botanif(t)e Publifum mit einer beffern und beutlichern 2(bbildung noch) zu bef(benEer.

19) Derglcichen Beipipiele beobachtete $\mathfrak{2}$ bu Iichen Aftrifa. 


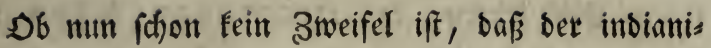

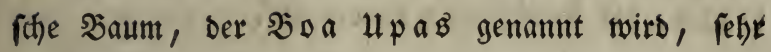
giftig ley, to werben bod) werfdiebene aberglaubifhe, unb Eeinen હ́lauben verbienende umftånbe von bem:

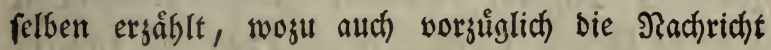
gebort, bie ein gewiffer bollánbifher Schiffwumbarst,

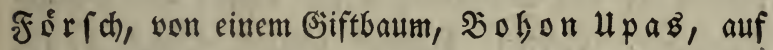
Java, mittheilt, und weldhe Nachridht berfelbe in bie Algem. Vaderl. Letteroefeningen Zesde Deel Tweede Stuk 1784. einrudfen laffen, aus wel: c)em 2 Serfe fie hernach in bas Gentleman's Magazin, und in bas \{eipsiger Nagazin zur Nathematif, Siaturlehre und Defonomie, Jabrgang I784, Des: Bleichen im Efprit des Journaux Année I785 mois de Juin, in Den Giơttingifhen Tajhenfalen: ber won eben biefem Jafr, unb fobann auch in ben vierten ફans ber Onomatologia medico - practica aufgenommen morben iff.

Diefe Nachridht hatte fo viel Fabelhaftes an fid,

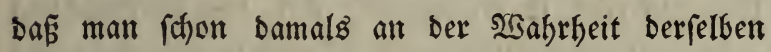
- jmeifelte. Gelbft in Sftinbien, nobin baz Sthef ber Letteroefeningen, weldyes biefe গachridht entfielt, fam, war biefelbe nell. Ilm aber vóllig Ginter ben Grumb ober lngrund berfelben ju fommen, nabm es die (jeit I778 errichtete) Gejellichaft Der Wiffen: (d)aften zu Satavia ůber fid), bie wabre befhaffen, beit biefer Sache zu unterfuchen, und trug babero 
ifren Mitgliebern, ben Scerren van $\Re$ gy $\mathfrak{P a} \mathfrak{l}$, bie 2 lusfůtrung bavou auf.

Die Refultate ihrer Bemulhungen finbet man in Nieuwe Algem. Vaderl. Letteroefeningen Vierde Deel, Tweede Stuk 1789. S. 104. ff. wovon in Den Sammlungen zur \$rypfit und Natur= geidjichte, vierten bantes viertes Stůf 1790.

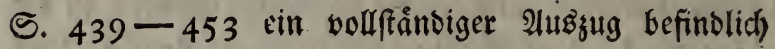
ift.

(Sleid) in 2 nfange biefer Schrift befinden fid) zwei Sriefe, wowon ber cinewan den Saifer $\mathfrak{P a c c o}$ bua a a Den Dritten, in Ştra=Sarta auf Java von

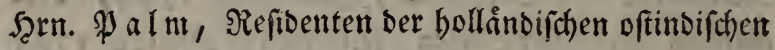
Sompagnie bei bemelbetem Rinifer, unter-ben 12, No: vember I785. in ber Feftung De \$offenburg, gefdries

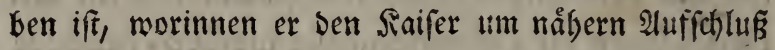
bittet, und fich erkundigt, in wie weit jene Siachricht

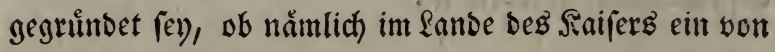

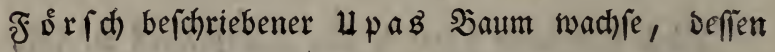
Bift auf eine fo ceremoniclle Int von Miffetbåtern

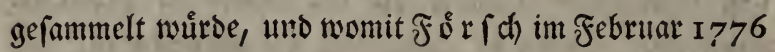
innerbalb ben Sabaton (innern f̧of) Der faiferl. গRe fibells Dreigebn Seifdlafferinten beb Raifers, bie ber Untreue gegen benjelben liberfilget worben, burds eimen fleinen Stich in bie Stuft, vermittelft eines mit ben upas vergifteten Sinftruments, innerhalb (ech)sebn Nimuten babe bincid)ten feben แ. f. m. 


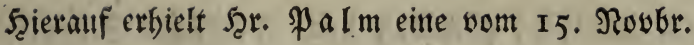
eben beffelbigen Jahrs, mit bem faiferlichen Siegel verfeheme, uno burdh Den Sufubunangh gefdriebene Antwort, in welcher ber Raijer gang furs erwiebert,

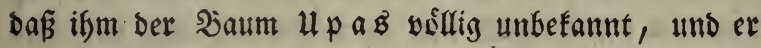
nie Das geringfte Dawon geheirt babe ${ }^{20}$ ).

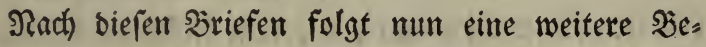
leuchtung ber offenbaren $\mathfrak{W}_{3}$ iberfpruche uns unwabr: beitest, welcher fich) Fo̊r (d) bei feiner (Ergåglung be= bient hat, und bie theils ber geographifchen lage eis niger Plåşe, ber Juftiţverfaffung, ben Bebråuchen und Sitten ber Şolfer auf Java, Der bei benfelben eingefurterten Sobesftrafen, und theils ber vom upas berrihgren follenden Ungefundbeit bes gangen Eilan. Des, offenbar mideripridyt.

Dá̃ biefer Saum auf 2Amrathen bes \$ropheten Mohammed, bie Suinden jul frafen, von Gott hervor:

$\mathfrak{g e s}$

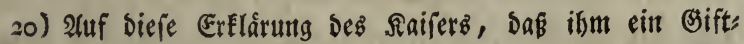
baum angefúlynter strt in feinem Sanbe gams unbefantut fey, war wobl nicft suverlábig su bauen: Denn ba man

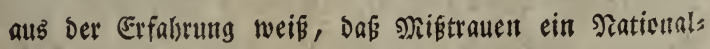
farafter biefer solfer iff, fo ware fich befonders auch Daruber nicht zat verwundern, wenn fie bas Dafenn eines Probuft berbeimlichten, womit fie in Sriegşseiten ibren Seinden gefábrlich werben fọnner. 


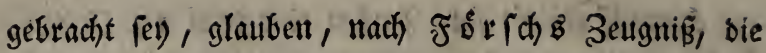
Mobammedaner beilig: jefir wafridjeinlid) mag bies fes aber eime fegende ber liftigen mobammebanifhen

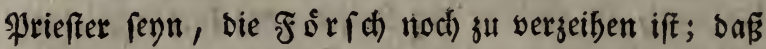
aber in ber Segend von Dem Baume auf zebn bis z'molf Meilen fein Pflångchen wachpe, unb in einem nod) weitern Umfreis von funfzelyn bis achtzebn Neis

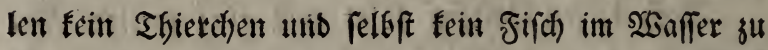

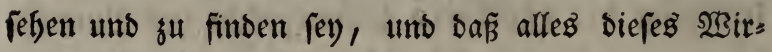
futrgen beb Giftbaumb wåren, håtte er, als ein aufs

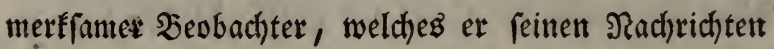
zil folge boch fenn mill, nicht für fuverlåfig unto wabl auşgeben follen. Dẩ man allenfalls, wie oben angefuigrt worben ift; sie auf cine Strecfe unt Den Saum berum befindliche Unfruchtbarkeit bes $\mathfrak{b}$ benz, ben giftigen 2 A ben tơnne, faun einigen Ģlauben verbienen, aber és

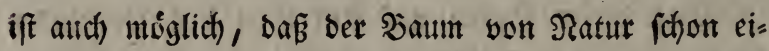
nen io unfuchtbaren Soben liebe, bem in Afrifa

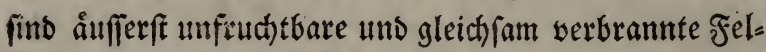

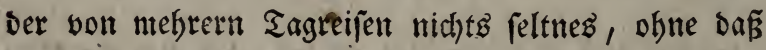

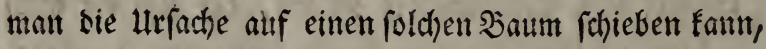
unb it ben trodfenften und wuifen Gegenden biefes Siselttheils fann ein aufmerffamer Sieifenoer mefrere Monate binburd), in welchen Fein einsiger Tropfen Regent ober Thau fất, fein Thierchen entbecken, $\Delta b=$ (thon in ben vier ůbrigen Nonaten, wo meiffentbeils siegenmetter ift, megrere wilde Sfiere, und felbft bie 


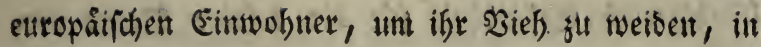
biefé G̈egenden jiehen.

Eine grobe thmabrheit if es, went $\bar{F}$ of $x$ id)

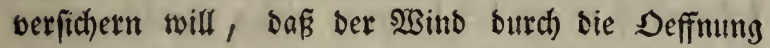
in Der Sette von Siergen, welche Die Giegeno unt Den Saum herum einfhliefen foll, bas Gift auf bev gansen Jufel verbreite, und biefelbe fo ungefund ma: che. Der grópte Iheil von Java if sielmefr, nach bem 3eugniffe glaubmuirbiger Reifenden, eineb ser gefundeften und fruchtbarften fănder in ser Şelt; und

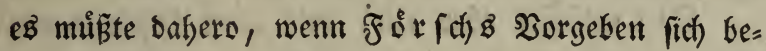
ftaittigen follte, aud) biefe vergiftete Ruft auf bas

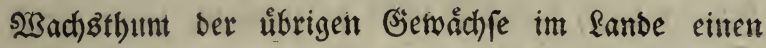
grofenen Einflup baben: fo aber jeigen vielmebr sie

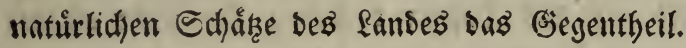

IIfentyalben bedecten biêés vorttefliche cilans Sie fdoonften Reifffelder, mit abwect)felnsen fruchtba=

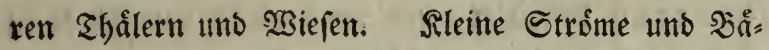
d)e, bie fum Theil von ber Sonme gleichfam verfil= bert, mit angenefmen Geråuld zwifd)en sen bunten (sebirgen herabfallen, burdmâffern bas fanb eines beftandigen Sommers, und gereichen bem Raffeebaum, Dem Bufferrobr und ber Indigpflange zum eritif den=

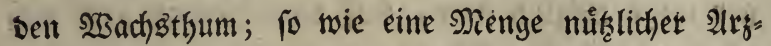
neigemåd) und Epezereien burch ifren buftenden $230 h l_{1}$ 
23oblgenth) Gefiunbheit für bie belebte Natur vera breitet.

Nur allein bie Ģegent um Sbatabia berunt, ift befanntlid) wegen Der bortigen vielen Mioråfte, Deren 24usbuำfung bie Ruft verunteinigt, ungefund, unb bies fe verborbene \&uft erregt freilich, befonbers mit Scûls fe. Der bort gewoofnlid)en Scisce, in ben Rơrpern ber

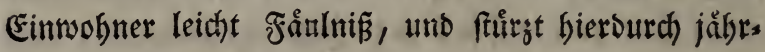
(id) eine Nenge Derfelben in bas Grab.

Dá̃ indeffen aber cin, wie oben befhriebener Saum, beffen Saft und Iusbuinftung to̊stlich ift, auf Java wadjpe, baran ift nidht jli zweifeln, wenn gleid) Die Scrn. $\mathfrak{P} a l m$ und $\mathfrak{v a} \mathfrak{n} \mathfrak{\Re} \mathfrak{h y} \mathfrak{n}$ in angefiugrter Sd)rift auf bas Dafenn beffelben fidh nid)t eigents

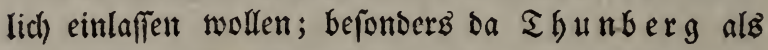
Der groófte Sotanift, ber je auf Java gewefen iff, bies pes unter antern im angefúhrten Srief an ben Sitter Murxay beftáttigt, und wo berfelbe bamals ichon

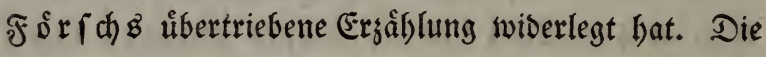

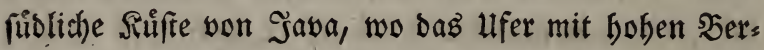
getr, ungehettern $\mathfrak{B a ̊ l b e r n , ~ E i n u o b e n , ~ S i l i p p e n ~ u n o ~}$ fumpfichten Dertern verfegen ift, ift bis jesco nod' zu

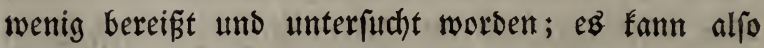
gar wohl mo̊glid feyn, bafi biefe Gegeno auch ser:

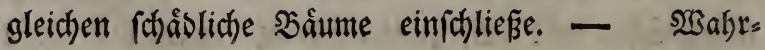
(c) ein= 
(h)einlid) fammelı bie Eingebohrnen bas Sift am meis fien nur zul Rrieggseiten, weil fie eg aufferbem wenis niger zu gebraudhen wiffen, bann buirfte $\mathfrak{e B}$ aber aud nicht etwas fo ungewoobnlid)es feyn, fich beffen sunt 3ergiften ber \$feile ju bebienen, ba aufierbem bie gefitteten Jnoianter Durd) Den ungang nit Europáern, Das Schiefigemebr fennen gelernt haben, Deffen Ses braud) fie jene vergifteten, und sffters fur fie felbft gefäbrlichen șaffen gerne vergeffen mad)t. 


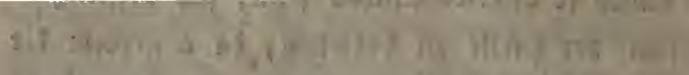

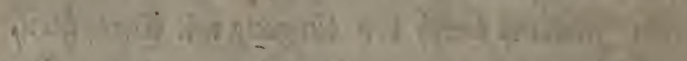

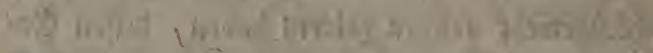

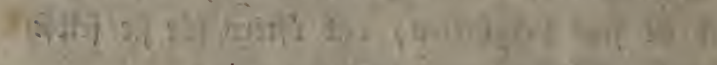

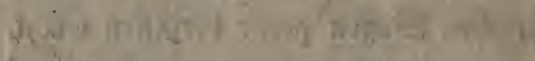
,

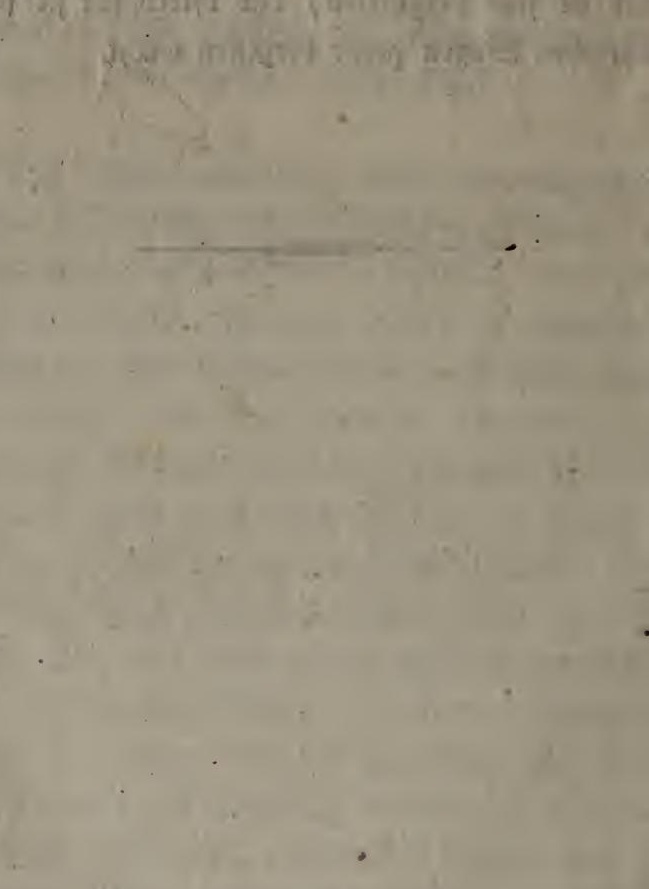




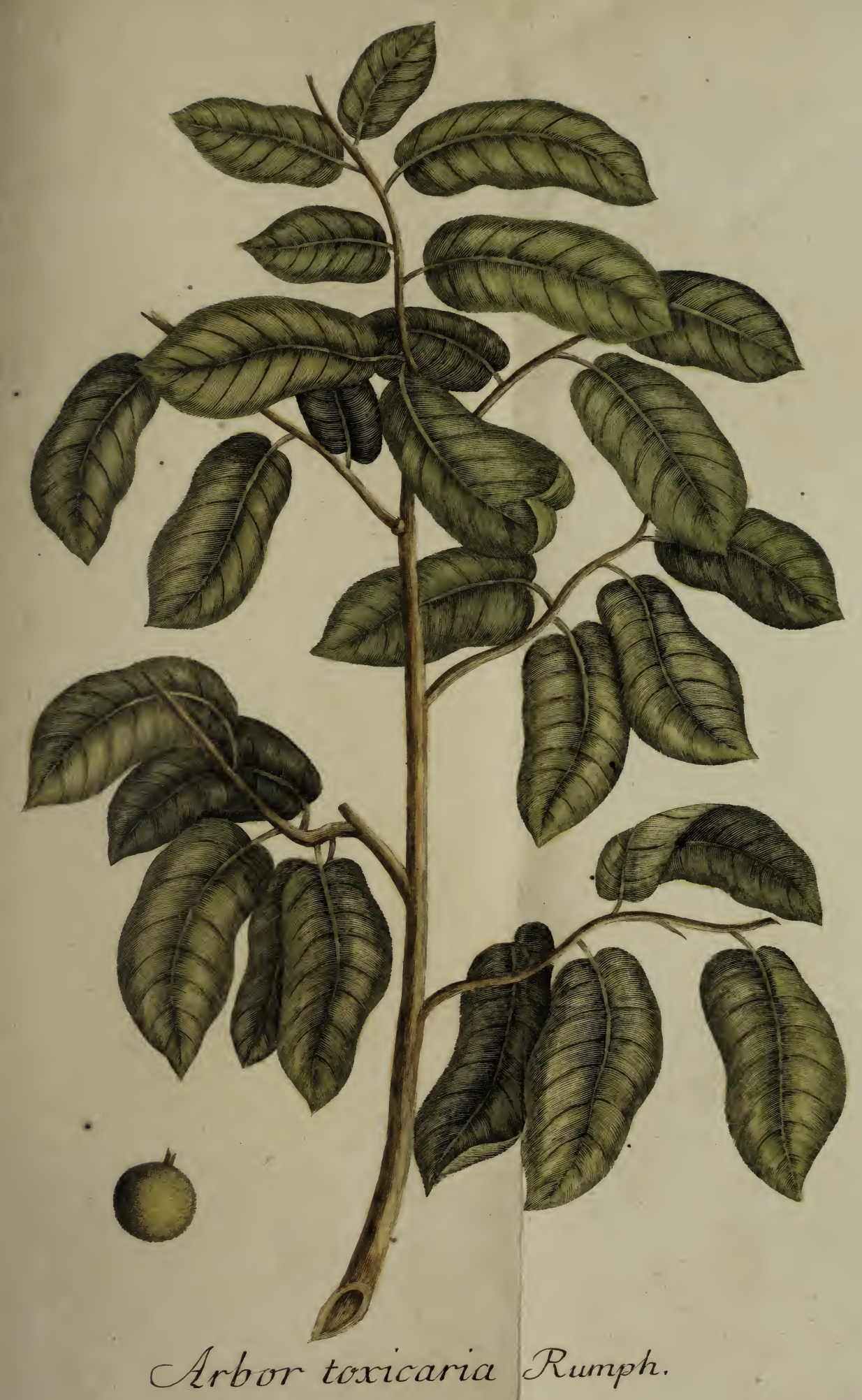





